\title{
A first estimate of triply heavy baryon masses from the pNRQCD perturbative static potential
}

\author{
Felipe J. Llanes-Estrada ${ }^{a}$, Olga I. Pavlova, Richard Williams \\ Departamento de Física Teórica I, Universidad Complutense, 28040 Madrid, Spain
}

Received: 16 December 2011 / Revised: 20 April 2012 / Published online: 3 June 2012

(C) Springer-Verlag / Società Italiana di Fisica 2012

\begin{abstract}
Within pNRQCD we compute the masses of spin-averaged triply heavy baryons using the now-available NNLO pNRQCD potentials and three-body variational approach. We focus in particular on the role of the purely threebody interaction in perturbation theory. This we find to be reasonably small and of the order $25 \mathrm{MeV}$. Our prediction for the $\Omega_{c c c}$ baryon mass is 4900 (250) MeV in keeping with other approaches. We propose to search for this hitherto unobserved state at $B$ factories by examining the end point of the recoil spectrum against triple charm.
\end{abstract}

\section{Introduction}

Amongst the staples of hadron physics is baryon spectroscopy. Here, quark model computations of the light baryon spectrum [1-3] find only mild success beyond ground-states in various channels due to the plethora of open thresholds and couplings between channels. A much cleaner system can be provided by baryons composed of three heavy quarks (i.e. the combinations $c c c, b b b, c c b$ and $b b c$ ). Since a considerable number of states are supposed to be below any strong decay thresholds, one can straightforwardly apply few-body reasoning, and quark model techniques can handle bound states better.

Indeed, a review of the literature reveals many studies that have computed or constrained heavy baryon masses, particularly the ground state $\Omega_{c c c}$. We collect some of the values obtained in Sect. 7 below. Like its meson (quarkonium) counterpart $[4,5]$, we expect this triply heavy baryon to attract much interest.

For heavy quark systems, the development of potential Non-Relativistic Quantum Chromodynamics (pNRQCD) as an effective theory of QCD has allowed a more systematic treatment of the theoretical uncertainties involved in spectroscopic predictions by expanding in powers of $1 / m$ [6-8].

a e-mail: fllanes@fis.ucm.es
For those in their ground state, pNRQCD can additionally be organized in standard perturbation theory as a power expansion in $\alpha_{s}[6,9]$. While the theory itself has limitations due to the finiteness of the quark masses, the two-body static potential (quickly reviewed in Sect. 2) has shown to be a good starting point for many meson investigations.

With the three-body potential in NNLO perturbation theory now at hand [10] (we will give it in Sect. 3), it is timely to perform an exploratory study of the ground-state triply heavy quark spectrum. This we present in Sect. 8. The necessary QCD parameters $\alpha_{s}, m_{c}, m_{b}$ are fixed by describing several common meson spectroscopy observables as explained in Sect. 5.

Finally, we comment on the feasibility of detecting the $\Omega_{c c c}$ in Sect. 10. Some numerical methods are relegated to Appendices A and B.

\section{Static quarkonium potential in pNRQCD}

The static two-body potential for bound states of a quark and anti-quark is well known to NNLO (and beyond) [11-13] as

$V^{(0)}=V_{\mathrm{LO}}^{(0)}+V_{\mathrm{NLO}}^{(0)}+V_{\mathrm{NNLO}}^{(0)}+\cdots$.

The leading order potential is just the color Coulomb potential

$V_{\mathrm{LO}}^{(0)}=-\frac{4}{3} \frac{\alpha_{s}\left(r^{-2}\right)}{r}$

whereas the NLO and NNLO are, respectively,

$$
\begin{aligned}
V_{\mathrm{NLO}}^{(0)}= & V_{\mathrm{LO}}^{(0)} \times \frac{\alpha_{s}\left(r^{-2}\right)}{4 \pi} \times\left(a_{1}+2 \gamma_{E} \beta_{0}\right), \\
V_{\mathrm{NNLO}}^{(0)}= & V_{\mathrm{LO}}^{(0)} \times \frac{\alpha_{s}^{2}\left(r^{-2}\right)}{(4 \pi)^{2}}\left(\gamma_{E}\left(4 a_{1} \beta_{0}+2 \beta_{1}\right)\right. \\
& \left.+\left(\frac{\pi^{2}}{3}+4 \gamma_{E}^{2}\right) \beta_{0}^{2}+a_{2}\right) .
\end{aligned}
$$


By standard convention $\gamma_{E} \simeq 0.57721 \ldots$ is the EulerMascheroni constant; for three colors and in terms of the number of quark flavors $N_{f}$ below the renormalization scale, the beta function that determines the running of the coupling is expanded as

$\beta_{0} \equiv 11-\frac{2 N_{f}}{3}$,

$\beta_{1} \equiv 102-\frac{38 N_{f}}{3}$,

and the coefficients in the potential that remain in a conformal theory are

$$
\begin{aligned}
a_{1} \equiv & \frac{31}{3}-\frac{10 N_{f}}{9}, \\
a_{2} \equiv & \frac{4343}{18}+36 \pi^{2}-\frac{9 \pi^{4}}{4} \\
& +66 \zeta(3)-\left(\frac{1229}{27}+\frac{52}{3} \zeta(3)\right) N_{f}+\frac{100}{81} N_{f}^{2},
\end{aligned}
$$

where $\zeta(3) \simeq 1.202 \ldots$ is Riemann's zeta function.

The NLO potential can be understood not only from pNRQCD (first, construct an effective theory around the heavy quark limit, then use Coulomb gauge in intermediate steps to obtain the gauge-invariant potential that is a matching coefficient in pNRQCD) but also from the $D_{\sigma \sigma}(|\mathbf{q}|)$ time-like gluon propagator obtained in Coulomb gauge by Watson and Reinhardt [14, 15], reducing it to its simpler heavy quark limit (there, several terms do not contribute to the Wilson loop potential). ${ }^{1}$

In momentum space, the potential can be given to order NNLO [12] at an arbitrary renormalization scale $\mu^{2}$ as

$$
\begin{aligned}
V\left(q^{2}\right)= & -\frac{4}{3} \frac{(4 \pi) \alpha \overline{M S}}{\mathbf{q}^{2}}\left(1+\frac{\alpha \overline{M S}}{4 \pi} C_{1}^{\overline{M S}}\left(\frac{\mu^{2}}{q^{2}}\right)\right. \\
& \left.+\left(\frac{\alpha \overline{M S}}{4 \pi}\right)^{2} C_{2}^{\overline{M S}}\left(\frac{\mu^{2}}{q^{2}}\right)\right)
\end{aligned}
$$

with

$$
\begin{aligned}
& C_{1}^{\overline{M S}}(x)=a_{1}+\beta_{0} \log (x), \\
& C_{2}^{\overline{M S}}(x)=a_{2}+\beta_{0}^{2} \log ^{2}(x)+\left(\beta_{1}+2 \beta_{0} a_{1}\right) \log (x) .
\end{aligned}
$$

This obviously simplifies if the renormalization scale is chosen as $q^{2}$ itself

$$
\begin{aligned}
V\left(q^{2}\right)= & -\frac{4}{3} \frac{(4 \pi) \alpha_{\overline{M S}}\left(q^{2}\right)}{\mathbf{q}^{2}} \\
& \times\left(1+a_{1} \frac{\alpha_{\overline{M S}}\left(q^{2}\right)}{4 \pi}+a_{2}\left(\frac{\alpha_{\overline{M S}}\left(q^{2}\right)}{4 \pi}\right)^{2}\right) .
\end{aligned}
$$

${ }^{1}$ We thank Jacopo Ghiglieri for this observation.

\section{$2.11 / m$ potential}

In QED the $1 / m$ corrections to the static potential were long ago shown to vanish. ${ }^{2}$

In perturbative $\mathrm{QCD}$ the non-Abelian vertex correction produces a $1 / m$ potential that cannot be gauged away. It can be nominally assigned to the $1 / \mathrm{m}^{2}$ order through a field redefinition [7]. Since a recent study of the meson spectrum $[17,18]$ finds reasonably large effects for the $1 S$ states, especially in charmonium, we also consider it here.

At leading order, the $1 / \mathrm{m}$ potential vanishes. For NLO and NNLO we employ the convention of [19]. Alternatively, one can use the NLO result (see Eq. (11) below) without [7] the factor $(7 / 9)$, in order to match a lattice computation.

In coordinate space the potential reads

$$
\begin{aligned}
V_{m^{-1}}= & -\frac{\alpha_{s}^{2}(\mu)}{m_{r} r^{2}} \times\left(\frac{7}{9}\right) \\
& -\frac{\alpha_{s}^{3}(\mu)}{3 \pi m_{r} r^{2}}\left\{-b_{2}+\log \left(e^{2 \gamma_{E}} \mu^{2} r^{2}\right)\left(\frac{7 \beta_{0}}{6}+\frac{68}{3}\right)\right\},
\end{aligned}
$$

where $m_{r}$ is the reduced (pole) mass of the $q \bar{q}$ system, $b_{2} \simeq-20.836$ for $N_{f}=3$ (appropriate for charmonium) and $b_{2} \simeq-18.943$ for $N_{f}=4$ (appropriate for bottomonium) and $b_{2} \simeq-17.049$ for higher scales where $N_{f}=5$ are given in [19]. The last term with a logarithm vanishes if the scale is chosen as the BLM scale defined by Eq. (A.2) in Appendix A. We have performed computations with both this running scale and a fixed scale $\left(m_{c}^{2}\right.$ or $\left.m_{b}^{2}\right)$.

If the potential is constructed in momentum space, the $1 / m$ correction to the central static potential reads

$$
\begin{aligned}
V_{m^{-1}}= & -2 \pi^{2} \frac{\alpha_{s}^{2}\left(\mu^{2}\right)}{m_{r} q} \times\left(\frac{7}{9}\right) \\
& -\frac{2 \pi \alpha_{s}^{3}\left(\mu^{2}\right)}{3 m_{r} q}\left(-b_{2}+\log \left(\frac{\mu^{2}}{q^{2}}\right)\left[\frac{7 \beta_{0}}{6}+\frac{68}{3}\right]\right) .
\end{aligned}
$$

Again, judicious choice of the scale $\mu=q$ disposes of the logarithm.

A counterintuitive result is that matrix elements of the $V_{m^{-1}}$ potential can actually be similar or, in extreme cases, even larger for bottom systems than for charm systems, since in a Coulombic system all energies scale with the reduced mass $m_{r}$.

\footnotetext{
${ }^{2}$ For example, in [16] it is shown that the Bethe-Salpeter ladder approximation generates Feynman gauge $1 / m$ terms that vanish upon including the crossed-ladder box. No such terms are present in Coulomb gauge.
} 
To see this, let us restrict ourselves to NLO and employ the convention of [7] in momentum space

$V_{m^{-1}}=-2 \pi^{2} \frac{\alpha_{s}^{2}\left(\mu^{2}\right)}{m_{r} q}$,

and compute $\left\langle\psi\left|V_{m^{-1}}\right| \psi\right\rangle$ with a hydrogen like wave function. Taking the Fourier transform of a $1 S$ state $2 e^{-r / a_{0}} /$ $\sqrt{4 \pi a_{0}^{3}}$, with Bohr radius $a_{0}^{-1}=m_{r} \alpha_{s}$ yields

$\psi(q)=\frac{4 \sqrt{4 \pi a_{0}^{3}}}{\left(1+q^{2} a_{0}^{2}\right)^{2}}$,

and therefore

$\left\langle V_{m^{-1}}\right\rangle=\frac{-2 a_{0}^{3}}{m_{r} \pi^{3}} \int \frac{d^{3} k d^{3} q}{|\mathbf{k}-\mathbf{q}|} \frac{\alpha_{s}^{2}(|\mathbf{k}-\mathbf{q}|)}{\left(\left(1+q^{2} a_{0}^{2}\right)\left(1+k^{2} a_{0}^{2}\right)\right)^{2}}$.

Extracting the dimensions and coupling, substituting the Bohr radius, and not minding about two constant positive numerical coefficients $c, c^{\prime}$, we find

$\left\langle V_{m^{-1}}\right\rangle=-m_{r} \alpha_{s}^{4}\left(\alpha_{s} m_{r} c\right) c^{\prime}$.

If the coupling constant did not run, the expectation value (though suppressed in the perturbative counting) would be some factor of $m_{b} / m_{c} \simeq 3$ larger for bottom than for charm systems. The NLO running of $\alpha_{s}$ however tames this growth and we will find an essentially constant behavior in the three-body computation (Fig. 12 below) as a function of quark mass between charm and bottom.

To give an example let us take pole quark masses of $m_{c}=$ $1.95 \mathrm{GeV}, m_{b}=5.14 \mathrm{GeV}$ and $\alpha_{s}\left(m_{Z}\right)=0.114$ (various fits of these quantities will be given later on in Sect. 6, these serve as illustration).

At the soft scale, with reduced quark mass $\frac{m}{2}$, solving iteratively as shown in Sect. B.3, $\alpha_{s}\left(\frac{m_{c}}{2} \alpha_{s}\right)=0.625$ and $\alpha_{s}\left(\frac{m_{b}}{2} \alpha_{s}\right)=0.395$. Then $\alpha_{s}^{4}(c) / \alpha_{s}^{4}(b) \simeq 6.3$. If the constant $c$ in Eq. (16) is somewhat smaller than one, this number could be smaller and around three.

\subsection{Running of the strong coupling constant}

The renormalization group equation that determines the running of the strong coupling constant to NNLO is

$\frac{\partial \alpha_{s}}{\partial \log q^{2}}=-\frac{\beta_{0}}{4 \pi} \alpha_{s}^{2}-\frac{\beta_{1}}{(4 \pi)^{2}} \alpha_{s}^{3}$.

By keeping the first term on the right hand side, or both terms, this equation can be solved to NLO or NNLO respectively.

In general we employ the Runge-Kutta algorithm to numerically solve Eq. (17). To NLO the equation is also very simply analytically solvable and provides a handy check for
Table 1 Benchmarks for $\alpha_{s}\left(\mu^{2}\right)$ at various scales. Input is the value of the coupling at the $Z$ mass $[21,22]$. Shown are NLO and NNLO results with the number of active flavors decreased in a step-wise fashion from five to four and then three at each of the benchmark thresholds

\begin{tabular}{llll}
\hline$\mu(\mathrm{GeV})$ & $N_{f}$ & NLO & NNLO \\
\hline 91.188 & 5 & $0.1184(7)$ & $0.1184(7)$ \\
5 & 4 & $0.204(2)$ & $0.2136(6)$ \\
1.6 & 3 & $0.295(4)$ & $0.336(2)$ \\
0.8 & - & $0.417(8)$ & $0.574(7)$ \\
\hline
\end{tabular}

the computer programme. Following [20] we introduce a scale $\Lambda$ as is customary, so that

$\alpha_{s}^{\mathrm{NLO}}\left(Q^{2}\right)=\frac{1}{b \log \frac{Q^{2}}{\Lambda^{2}}}$,

with

$b=\frac{\beta_{0}}{4 \pi}=\frac{33-2 N_{f}}{12 \pi}$.

Inverting the equation yields

$\Lambda^{2}=Q^{2} \exp \left(-\frac{1}{b \alpha_{s}^{\mathrm{NLO}}\left(Q^{2}\right)}\right)$.

In Table 1 we give for convenience, and as benchmarks, the values obtained by running back to low scales the renormalization group equation from the Z-boson pole, where the coupling constant is very accurately constrained by many analyses [21, 22]. At each of the scales mentioned in the table the number of active flavors in the beta function is decreased by one in a stepwise fashion and continuous matching is performed. ${ }^{3}$ A typical run in agreement with world average and low-scale $\tau$ data is plotted in Fig. 1. In addition we employ a second coupling that provides a best fit to key charmonium and bottomonium data, which, while still broadly consistent with high energy data, are somewhat smaller.

Moreover, we vary the number of flavors dynamically in the computer programme upon crossing each threshold. Since the quark masses themselves are varying during each fit we cannot quote these thresholds here. However, they are in the vicinity of $1.6 / 1.7 \mathrm{GeV}$ for the charm and $5 \mathrm{GeV}$ for the bottom threshold.

We know that the value of $\alpha_{s}$ at the Z-boson pole, evolved by backward NNLO running, is consistent with the very precise determination based on radiative decays of the $\Upsilon$ meson [24], $0.184 \pm 0.015$ at the bottomonium scale. Therefore, deviations from the Z-pole value in our fit results give

\footnotetext{
${ }^{3}$ This introduces a non-analyticity that can, at least at NLO, be avoided by the use of the Brodsky-Lepage-Mackenzie method. See Appendix A. This non-analyticity is enhanced if one employs the discontinuous matching conditions [23] based on effective theory, which we also intend to incorporate in future work.
} 


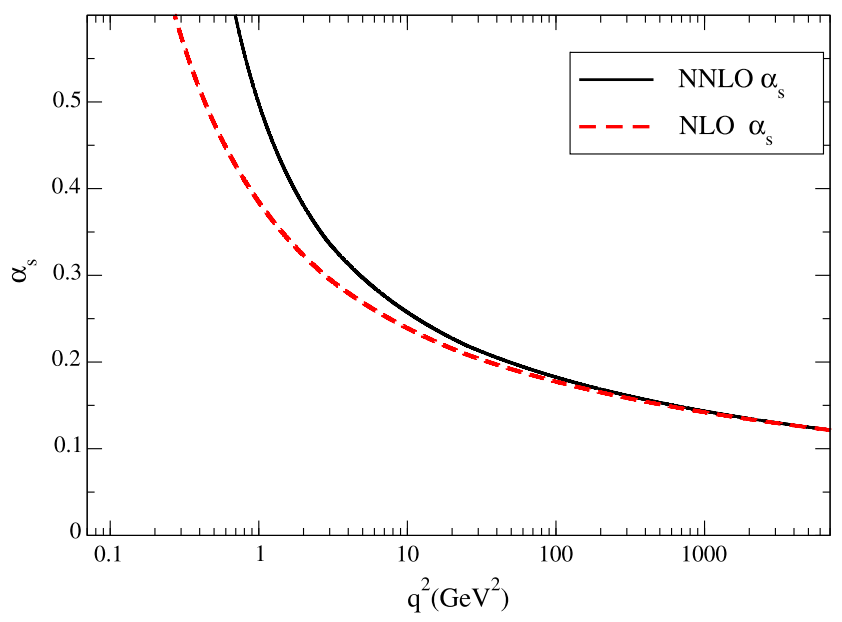

Fig. 1 Typical running coupling constant to order NNLO agreeing with recent $\tau$ data and the world average [21,22]

us an idea of the size of the errors associated with the heavyquark effective potentials. No further perturbation-theory approximation in the computed masses is implicit, given that the Schroedinger equation is exactly diagonalized.

Finally, we remark that infinitely heavy quarkonium should be less sensitive to the infrared details of the interaction, but that some sizeable sensitivity remains because the quark mass is finite. Thus we employ the heavy quarkpotential for all $r$ in the programme. To avoid encountering spurious Landau pole singularities, we freeze the coupling constant at a low scale (400-600 MeV) and check the sensitivity to this procedure below.

In conclusion, each of the runs reported will employ a slightly different fit value of $\alpha_{s}$, but typical shapes for the coupling constant can be seen in Figs. 1 and 2.

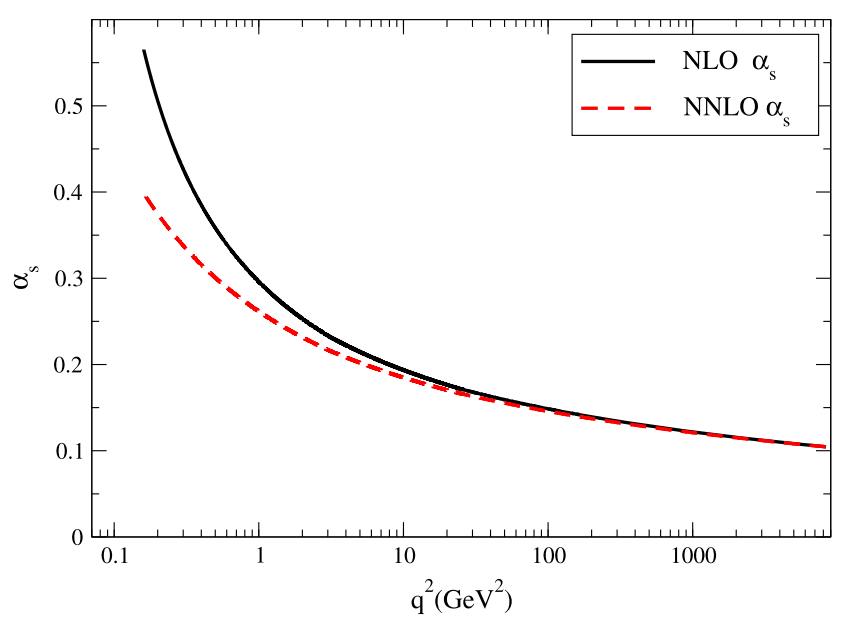

Fig. 2 Typical running coupling fit to two-body data. The actual best fit scale will depend on the order of perturbation theory, possible renormalon subtraction and treatment of the infrared, whether the static potential is or is not corrected by the $1 / \mathrm{m}$ force, etc., and varies from computation to computation as will be indicated below

\section{Heavy baryon potential in perturbation theory}

The static potential between three heavy quarks of equal mass in positions $\mathbf{r}_{1}, \mathbf{r}_{2}, \mathbf{r}_{3}$ is expanded in powers of the strong coupling constant as

$V^{(0)}\left(\mathbf{r}_{1}, \mathbf{r}_{2}, \mathbf{r}_{3}\right)=V_{\mathrm{LO}}^{(0)}+V_{\mathrm{NLO}}^{(0)}+V_{\mathrm{NNLO}}^{(0)}+\cdots$.

In this work we will obtain the masses of a few heavy baryons from the leading order and next to leading order potentials by employing a variational basis. Then we will study the effect of the intrinsic three-body force (star-shaped) that appears first at NNLO, and finally employ the rest of the known two-body NNLO terms to estimate further corrections to the mass values.

The leading order potential is $\Delta$-shaped i.e. given by the sum of the two-body Coulomb interactions

$V_{\mathrm{LO}}^{(0)}=\frac{-2 \alpha_{s}}{3}\left(\frac{1}{\left|\mathbf{r}_{1}-\mathbf{r}_{2}\right|}+\frac{1}{\left|\mathbf{r}_{2}-\mathbf{r}_{3}\right|}+\frac{1}{\left|\mathbf{r}_{3}-\mathbf{r}_{1}\right|}\right)$.

For the remainder of this section we will shorten the notation by summing over an index $i=1,2,3$ that runs over the three possible pairings of the quarks, so that

$V_{\mathrm{LO}}^{(0)}=\frac{-2 \alpha_{s}}{3} \sum_{i} \frac{1}{\mathbf{r}_{i}}$

The one-loop corrections to this potential yield the NLO part. The coupling constant is renormalized and one needs to choose the renormalization scale at which the constant is initially given. Following the pNRQCD custom, we first select the renormalization scale $\mu_{i}^{2}=1 /\left|\mathbf{r}_{i}\right|^{2}$. Then the potential to NLO [10] reads

$$
\begin{aligned}
V_{\mathrm{LO}}^{(0)}+V_{\mathrm{NLO}}^{(0)}= & -\frac{2}{3} \sum_{i} \alpha_{s}\left(\left|\mathbf{r}_{i}\right|^{-2}\right) \frac{1}{\left|\mathbf{r}_{i}\right|} \\
& \times\left[1+\frac{\alpha_{s}\left(\left|\mathbf{r}_{i}\right|^{-2}\right)}{4 \pi}\left(2 \beta_{0} \gamma_{E}+a_{1}\right)\right] .
\end{aligned}
$$

In momentum space, the NLO potential is easily reconstructed by comparison with Eq. (10).

We now turn to the potential at NNLO. We are first of all interested in the intrinsic three-body piece, the star-shaped part of the potential that appears at this order. A typical Feynman diagram is represented in Fig. 3. While three-body forces have been considered in the context of heavy hybrid mesons [25], applications to triply heavy baryons are sparse.

This three-body force is conveniently organized in terms of an auxiliary potential

$$
\begin{aligned}
V_{\mathrm{NNLO}-3}^{(0)}= & 2\left(V_{\mathrm{aux}}\left(\mathbf{r}_{2}, \mathbf{r}_{3}\right)+V_{\mathrm{aux}}\left(\mathbf{r}_{1},-\mathbf{r}_{3}\right)\right. \\
& \left.+V_{\mathrm{aux}}\left(-\mathbf{r}_{2},-\mathbf{r}_{1}\right)\right)
\end{aligned}
$$




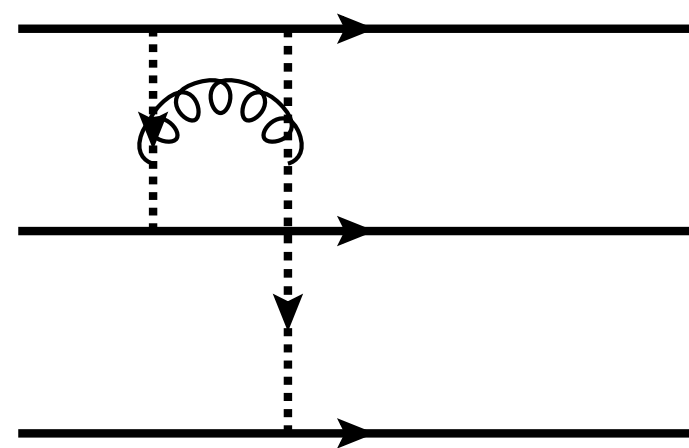

Fig. 3 One of 12 Feynman diagrams contributing to the intrinsic threebody force in a triply heavy baryon at NNLO

which is computed via the Fourier transform

$V_{\text {aux }}\left(\mathbf{r}_{2}, \mathbf{r}_{3}\right)=i \int \frac{d \mathbf{q}_{2} d \mathbf{q}_{3}}{(2 \pi)^{6}} e^{i \mathbf{q}_{2} \cdot \mathbf{r}_{2}} e^{i \mathbf{q}_{3} \cdot \mathbf{r}_{3}} \hat{V}_{\text {aux }}\left(\mathbf{q}_{2}, \mathbf{q}_{3}\right)$

of the potential

$$
\begin{aligned}
\hat{V}_{\text {aux }}\left(\mathbf{q}_{2}, \mathbf{q}_{3}\right) \\
=\frac{(-i / 2)(4 \pi)^{3} \alpha_{s}^{3}}{8\left|\mathbf{q}_{2}\right|^{2}\left|\mathbf{q}_{3}\right|^{2}}\left[\frac{\left|\mathbf{q}_{2}+\mathbf{q}_{3}\right|}{\left|\mathbf{q}_{2}\right|\left|\mathbf{q}_{3}\right|}+\frac{\mathbf{q}_{2} \cdot \mathbf{q}_{3}+\left|\mathbf{q}_{2}\right|\left|\mathbf{q}_{3}\right|}{\left|\mathbf{q}_{2}\right|\left|\mathbf{q}_{3}\right|\left|\mathbf{q}_{2}+\mathbf{q}_{3}\right|}\right. \\
\left.\quad-\frac{1}{\left|\mathbf{q}_{2}\right|}-\frac{1}{\left|\mathbf{q}_{3}\right|}\right] .
\end{aligned}
$$

The two momenta $q_{2}$ and $q_{3}$ are flowing out of the two quark lines.

The two-body ( $\Delta$-like) contribution to the potential at NNLO is a simple generalization of Eq. (4)

$$
\begin{aligned}
V_{\mathrm{NNLO}-2}^{(0)}= & -\frac{2}{3} \sum_{i} \frac{\alpha_{s}\left(\mathbf{r}_{i}^{-2}\right)}{\left|\mathbf{r}_{i}\right|} \frac{\alpha_{s}\left(\mathbf{r}_{i}^{-2}\right)^{2}}{(4 \pi)^{2}} \\
& \times\left(a_{2}-36 \pi^{2}+3 \pi^{4}+\left(\frac{\pi^{2}}{3}+4 \gamma_{E}^{2}\right) \beta_{0}^{2}\right. \\
& \left.+\gamma_{E}\left(4 a_{1} \beta_{0}+2 \beta_{1}\right)\right) .
\end{aligned}
$$

That ground state heavy baryons feel more of a two-body like $\Delta$-shaped rather than the $\Upsilon$-shaped potential is supported by lattice data, where the $\Delta$ ansatz seems dominant up to distances of $R \simeq 0.7 \mathrm{fm}$ [26] or even $1 \mathrm{fm}$ [27].

\section{Infrared subtracted potential}

The static potential in terms of the pole mass is afflicted by an infrared singularity in perturbation theory [28-30] that can be subtracted by a momentum-space cutoff [31]. In passing from momentum space to coordinate space this amounts to a restricted Fourier transform

$V_{\mathrm{PS}}\left(r, \mu_{f}\right)=\int_{|\mathbf{q}|>\mu_{f}} \frac{d^{3} q}{(2 \pi)^{3}} e^{i \mathbf{q} \cdot \mathbf{r}} V(|\mathbf{q}|)$.

Correspondingly, the quark mass one deduces from the fits to data is in this particular "Potential Subtracted" scheme (shortened "PS" in what follows). The infrared singularity (and accordingly, bad behavior of perturbation theory) is now exposed as a counterterm necessary if one wants to retrieve the pole mass

$m_{\mathrm{PS}}\left(\mu_{f}\right)=m+\frac{1}{2} \int_{|\mathbf{q}|<\mu_{f}} \frac{d^{3} q}{(2 \pi)^{3}} V(|\mathbf{q}|)$.

In perturbation theory, lacking another scale except the renormalization scale, the counterterm has to be proportional to $\mu_{f}$ up to logarithms, and is displayed explicitly in Eq. (34) below. This is so that the static energy of the system does not depend on the subtraction,

$V_{\mathrm{PS}}+2 m_{\mathrm{PS}}=V+2 m$.

One can avoid the singularity altogether by directly relating the PS mass with the $\overline{M S}$ mass by means of Eqs. (32) and (34).

The relation between pole quark masses $m$ and $\overline{M S}$ masses $\bar{m}$ to NNLO is given in terms of the number of light quarks $N_{f}$ as

$$
\begin{aligned}
m= & \bar{m}(\bar{m})\left[1+\frac{4}{3} \frac{\alpha_{s}(\bar{m})}{\pi}\right. \\
& \left.+\left(13.44-1.041 N_{f}\right)\left(\frac{\alpha_{s}(\bar{m})}{\pi}\right)^{2}\right],
\end{aligned}
$$

where $\alpha_{S}$ is understood as the $\overline{M S}$ coupling constant (in terms of which we have anyway expressed the heavy quark static potential). The inverse relation is [32]

$$
\begin{aligned}
\bar{m}(m)= & m\left[1-\frac{4}{3} \frac{\alpha_{s}(\bar{m})}{\pi}\right. \\
& \left.+\left(-14.33+1.041 N_{f}\right)\left(\frac{\alpha_{s}(\bar{m})}{\pi}\right)^{2}\right] .
\end{aligned}
$$

While using the Potential Subtraction scheme we need to convert the PS mass $m_{\mathrm{PS}}$ to the pole mass too. This is achieved by means of [31]

$$
\begin{aligned}
m= & m_{\mathrm{PS}}\left(\mu_{f}\right)+\mu_{f} C_{F} \frac{\alpha_{s}(\mu)}{\pi} \\
& \times\left(1+\frac{\alpha_{s}(\mu)}{4 \pi}\left[a_{1}-\beta_{0}\left(\log \left(\frac{\mu_{f}^{2}}{\mu^{2}}\right)-2\right)\right]\right) .
\end{aligned}
$$




\section{Meson observables}

If we handle the heavy charmonium and heavy bottomonium systems we need to fix three parameters, the strong coupling constant at one scale $\alpha_{s}\left(\mu^{2}\right)$ (the evolution equation of the renormalization group then provides it at any other scale) and the quark masses at their pole (or in any other scheme). Alternatively, we employ a fixed running coupling constant from Fig. 1 and fit only the quark masses to the ground state quarkonia (thus limiting ourselves to two parameters).

We will employ five observables to fix these parameters, two from charmonium ( $1 S$ mass and radiative transition $J / \psi \rightarrow \eta_{c} \gamma$ ), two from bottomonium ( $1 S$ and $1 P$ masses), and the $B_{c}$ mass. This will over constrain the parameters for additional security and allow us to check the running of the coupling between the two scales, at the charm and at the bottom.

Since the purely static potential does not accommodate a hyperfine $\mathbf{S}_{1} \cdot \mathbf{S}_{2}$, nor an $\mathbf{L} \cdot \mathbf{S}$ splitting, we will employ spin-averaged masses. For bottomonium, we employ the recently measured $\eta_{b}$ mass, 9391(3) MeV, and the $\Upsilon$ mass 9460.3(3) $\mathrm{MeV}$, whose spin average is $\left(M_{\eta_{b}}+3 M_{\Upsilon}\right) / 4=$ 9443(1) MeV. We also have at our disposal the $P$-wave mesons, which are also sometimes believed to lie in the regime where perturbative pNRQCD is applicable, $\chi_{b 0}$ with mass 9859.4(4) $\mathrm{MeV}, \chi_{b 1}$ with mass 9892.8(3) $\mathrm{MeV}$, and $\chi_{b 2}$ with mass $9912.2(3) \mathrm{MeV}$, which yield a spin average $\left(M_{\chi_{b 0}}+3 M_{\chi_{b 1}}+5 M_{\chi_{b 2}}\right) / 9=9899.9(3) \mathrm{MeV}$.

Looking towards the $2 S$ bottomonium levels (which, although their wave function starts being immersed in the nonperturbative part of the potential, can still to some approximation be considered perturbative quarkonium states), we notice that the $\eta_{b}(2 S)$ mass has not been measured yet, so that we take the $\Upsilon(2 S)$ as sole data, without the possibility of spin-averaging. The error introduced is expected to be of order $10 \mathrm{MeV}$ or less since the hyperfine splitting should be smaller than for the ground state (where the difference between the $\Upsilon$ and the spin average is $17 \mathrm{MeV}$ ). One should not expect this state to be well described in perturbation theory [33], but we show results nonetheless for comparison and completeness.

Turning to charmonium, the renowned $J / \psi$ state has a currently accepted mass of $3096.92(1) \mathrm{MeV}$, and the $\eta_{c}(1 S)$ a mass of 2980(1) MeV. Their spin averaged mass is therefore $\left(M_{\eta_{c}}+3 M_{J / \psi}\right) / 4=3067.7$ (3) MeV. The NRQCD description of the $2 S$ states in the charmonium system is generally not accepted, so to obtain one more observable at the charmonium scale we turn to the $S$-wave radiative transition width $J / \psi \rightarrow \eta_{c} \gamma$, decay branch $\Gamma_{133}$ of the $J / \psi$ in the notation of [34]. This branching fraction currently yields a width $\Gamma_{J / \psi \rightarrow \eta_{c} \gamma}=1.6(4) \mathrm{keV}$.

This radiative transition width between the $1 S$ charmonium states has also been calculated [35] at NNLO, and all we need to do is evaluate it numerically.
Table 2 Experimental data employed to fix the potential parameters (adapted from [34]). The error in parentheses refers to the last significant digit

\begin{tabular}{ll}
\hline$\left(M_{\eta_{b}}+3 M_{\Upsilon}\right) / 4$ & $9443(1) \mathrm{MeV}$ \\
$\left(M_{\chi_{b 0}}+3 M_{\chi_{b 1}}+5 M_{\chi_{b 2}}\right) / 9$ & $9899.9(3) \mathrm{MeV}$ \\
$M_{\Upsilon(2 S)}$ & $10023(0.3) \mathrm{MeV}$ \\
$\left(M_{\eta_{c}}+3 M_{J / \psi}\right) / 4$ & $3067.7(3) \mathrm{MeV}$ \\
$\Gamma_{J / \psi \rightarrow \eta_{c} \gamma}$ & $1.6(4) \mathrm{keV}$ \\
$M_{B_{c}}+(3 / 4) \Delta M_{h f}$ & $6317(8) \mathrm{MeV}$ \\
\hline
\end{tabular}

In addition we have at our disposal the mass of the $B_{c}$ meson, $M_{B_{c}}=6277(6) \mathrm{MeV}$. Here the vector $B_{c}^{*}$ is not yet known, so that the spin average has to be obtained by interpolation from other flavor combinations. Currently we can lean on the $B$ system for which the hyperfine splitting is $\Delta M_{h f}=45.8(3) \mathrm{MeV}$, the $B_{s}$ system where $\Delta M_{h f}=49(2) \mathrm{MeV}$, and the bottomonium $\Upsilon-\eta_{b}$ system where $\Delta M_{h f}=69(3) \mathrm{MeV}$. Our interpolated estimate for the $B_{c}$ flavor combination is therefore $\Delta M_{h f}=53(3) \mathrm{MeV}$. Adding this correction to the $B_{c}$ mass to yield a spin average $M_{B_{c}}+\frac{3}{4} \Delta M_{h f}$ gives $6317(8) \mathrm{MeV}$.

This parameter set is collected in Table 2.

\subsection{Evaluation of $\Gamma_{J / \psi \rightarrow \eta_{c} \gamma}$}

Here we comment on the numerical evaluation of the radiative transition between the two $1 S$ charmonium states, $J / \psi$ and $\eta / c$. At NNLO we read off [35]

$$
\begin{aligned}
\Gamma_{J / \psi \rightarrow \eta_{c} \gamma}= & \frac{16 \alpha_{\mathrm{em}}}{3} e_{c}^{2} \frac{k_{\gamma}^{3}}{M_{J / \psi}^{2}} \\
& \times\left(1+\frac{4}{3} \frac{\alpha_{s}\left(\left(M_{J / \psi} / 2\right)^{2}\right)}{\pi}\right. \\
& \left.-\frac{2}{3}\left(\frac{4}{3} \alpha_{s}\left(r^{-2}\right)\right)^{2}\right),
\end{aligned}
$$

where $e_{c}=2 / 3$ is the charm quark's charge in units of the electron charge, and the pole quark mass has been eliminated in terms of the physical $J / \psi$ mass. Since the experimental error in this observable is relatively large, it is not sensible to delve into subtleties about this elimination.

The momentum of the photon is given simply as $k_{\gamma}=$ $\left(M_{J / \psi}^{2}-M_{\eta_{c}}^{2}\right) /\left(2 M_{J / \psi}\right)$ by energy-momentum conservation.

The strong coupling constant is evaluated at two different scales in this formula, half the resonance mass (corresponding to the scale of the quark mass, or hard scale) and the momentum scale of the quark inside the resonance $p_{J / \psi}=r^{-1}$ (or soft scale). The first one is of direct evaluation, but since the characteristic momentum in charmonium 
is $\langle p\rangle \simeq m_{r} \alpha_{s}=\frac{m_{c}}{2} \alpha_{s}$, the second occurrence of the coupling constant has a scale that depends itself on the coupling constant, $\alpha_{S}\left(\frac{M_{J / \psi}}{4} \alpha_{s}\right)$.

To obtain $\alpha_{s}$ one then needs to employ Eq. (A.5) recursively. The method is detailed in Sect. B.3.

\section{Meson numerical results and parameter fixing}

\subsection{Exploratory fits}

In this subsection we gain a feeling for the parameter values and explore several alternatives. Table 3 presents the result of our fits numbered consecutively, not including the $B_{c}$ mass nor including the $1 / \mathrm{m}$ potential.

Fits 1, 2 and 3 show the consistency of perturbation theory, which is quite reasonable. In these fits the quark masses come out consistently in the $1.7 \mathrm{GeV}$ (charm) and $5 \mathrm{GeV}$ (bottom) ranges. Here $\alpha_{S}\left(m_{Z}^{2}\right)$, left free, varies more significantly upon increasing the order of perturbation theory, and is largely ensuring that the $1 P$ bottomonium level is in good agreement with experiment. In spite of this, the radiative $\psi \rightarrow \eta_{c}$ transition width is outside its experimental $2 \sigma$ error band, and the splittings in the bottomonium spectrum are significantly smaller than experiment. The smallness of this splitting pulls the spin-averaged $b \bar{b}(1 S)$ mass to higher than physical values.

The first of these defects is common to $c \bar{c}$ approaches, and within the present scheme it requires certain fine tuning. The second problem is related to the fact that bottomonium excitations start being sensitive to the linear part of the static potential, so that a purely perturbative quarkonium description is not very precise.

Fits 4 and 5 show the insensitivity to changing the freezing scale for the running coupling constant, which is fixed to $0.8 \mathrm{GeV}$, whereas it is 0.5 in all others. Comparing fits 3 and 4 for example, we see that the value of this constant is irrelevant for all purposes, except a marginal improvement in the bottomonium splittings.

Fit 5 is different in that the coupling constant is not allowed to vary, but fixed to the world average at the Z-pole. This larger value of the coupling brings the transition width to better agreement with experimental data. This is due to the NNLO contribution, negative, being much enhanced. The charm quark is however pushed to the limit of its variation band between 1 and $2 \mathrm{GeV}$ in the programme, and increases disagreement with other determinations.

In fits 6 and following we return to a freezing scale of $0.5 \mathrm{GeV}$ but change the way to compute the $\chi^{2}$ to be minimized. Instead of employing the experimental error bands $\sigma_{i \text { exp }}^{2}$ for the quarkonium masses in

$\chi^{2}=\sum \frac{\left(E^{\mathrm{th}}-E^{\mathrm{exp}}\right)_{i}^{2}}{\sigma_{i \exp }^{2}}$,

we adopt a common error band of $30 \mathrm{MeV}$ for all of them. This is in recognition that theory errors for these observables are orders of magnitude larger than experimental errors, and we want to check that the experimental errors are not weighing the various states unduly in the fit.

In fit 6 we separately fit the two charmonium observables and the three bottomonium observables, to ascertain the tension between them. This is visible from the two different

Table 2. The quark masses quoted are the pole masses and the $\overline{M S}$ masses at $3 \mathrm{GeV}$ to facilitate comparison
Table 3 Numerical results and fit parameters. Units are MeV except for the $(1 S)$ radiative decay width $\Gamma_{J / \psi \rightarrow \eta_{c} \gamma}$ which is quoted in $\mathrm{keV}$. The reference experimental data for the various fits can be found in

\begin{tabular}{|c|c|c|c|c|c|c|c|c|c|c|c|}
\hline Fit & Order & $m_{c \bar{c}}$ & $\Gamma_{J / \psi \rightarrow \eta_{c} \gamma}$ & $m_{b \bar{b}}(1 S)$ & $m_{b \bar{b}}(2 S)$ & $m_{b \bar{b}}(1 P)$ & $\alpha_{s}\left(m_{Z}^{2}\right)$ & $m_{c}$ & $m_{b}$ & $\bar{m}_{c}$ & $\bar{m}_{b}$ \\
\hline 1 & $\mathrm{LO}$ & 3068 & 2.7 & 9477 & 9836 & 9913 & 0.128 & 1720 & 4980 & 820 & 4600 \\
\hline 2 & NLO & 3068 & 3.0 & 9567 & 9808 & 9908 & 0.111 & 1740 & 5080 & 1160 & 4780 \\
\hline 3 & NNLO & 3068 & 2.6 & 9632 & 9820 & 9901 & 0.095 & 1700 & 5050 & 1330 & 4900 \\
\hline 4 & NNLO & 3068 & 2.6 & 9536 & 9806 & 9911 & 0.099 & 1720 & 5040 & 1340 & 4860 \\
\hline 5 & NNLO & 3068 & 1.9 & 9443 & 9813 & 9986 & $0.1184^{*}$ & $2000^{\dagger}$ & 5220 & 1350 & 4970 \\
\hline 6 & NNLO & 3070 & 2.4 & 9630 & 9830 & 9910 & $0.107 / 0.097^{\&}$ & $2000^{\dagger}$ & 5060 & & \\
\hline 7 & NNLO & 3067 & 2.4 & 9443 & 9548 & 9558 & 0.108 & $2000^{\dagger}$ & 5170 & 1530 & 4970 \\
\hline 8 & NNLO & 3067 & 2.6 & 9690 & 9824 & 9906 & 0.095 & 1700 & 5050 & 1368 & 4900 \\
\hline 9 & NNLO & 3068 & 0.74 & 9443 & 9685 & 9525 & 0.12 & 2188 & 5381 & 1540 & 5140 \\
\hline 10 & NNLO & 3068 & 1.6 & 9443 & 9688 & 9796 & 0.111 & 1853 & 5100 & 1340 & 4878 \\
\hline
\end{tabular}

${ }^{\dagger}$ At the limit of the allowed fitting range

*Fixed from the world average value at the Z-boson pole

\& Running coupling separately fit to the charm and bottom data 
values obtained for the coupling constant evolved to the Zpole, at the level of $10 \%$.

In fit 7 we leave the bottomonium $1 P$ excitation out of the $\chi^{2}$ formula to guarantee that the bottom quark mass is fixed to the bottomonium spin-averaged ground state. (It is included in other fits of this table, but generally not included in those of Sect. 6.2.)

We see that the width $\Gamma_{J / \psi \rightarrow \eta_{c}}$ pulls the charm quark mass again to the limit of our allowed variation band.

In fit 8 instead we decouple $\Gamma_{J / \psi \rightarrow \eta_{c}}$ from the minimization. This immediately relaxes back the charm quark mass.

In fit 9 we use as input the spin-averaged ground state masses of bottomonium and charmonium, together with the pseudodata result $\alpha_{s}\left(m_{Z}^{2}\right)=0.12$, which can be understood as a fit to the ratio of radiative to total widths of the $\Upsilon$ [24]. At the $\tau$ pole the running coupling is also in agreement with $\tau$ data, which suggests $\alpha_{S}(\tau)=0.330(25)$ [37]. The radiative width is now below the experimental value, showing that with fine tuning of the coupling constant it can be brought to the physical value. However, the $1 P$ bottomonium state is now very far off, due to the increased coupling, and the quark masses are far from other determinations.

We proceed to fit 10 , in which we force the reproduction of the precise experimental number for the $1 S$ radiative width of $1.6 \mathrm{keV}$. This happens for $\alpha_{S}\left(m_{Z}^{2}\right)=0.111$ at NNLO. As expected, this is possible at the expense of losing agreement with the $1 P$ mass.

Since the experimental error in the radiative width is so much larger than the error in the $1 P$ mass measurement, a best fit will try to compromise by lowering the coupling constant in spite of this deteriorating the computation of the width.

Overall it appears that to obtain a perfect value for $\Gamma_{J / \psi \rightarrow \eta_{c}}$ requires a little fine tuning, and that in no case is it possible to obtain an excellent fit to all five quantities simultaneously.

\subsection{Extended fits}

In this subsection we include the $B_{c}$ mass and explore in addition the effect of the $1 / m$ potential. In all cases the coupling constant is evolved to the $Z$ pole at NNLO (this should be for broad comparison and not taken as a detailed prediction since higher orders of perturbation theory should then be used).

Comparing Tables 4 and 5 we see that the effect of the $1 / m$ potential is modest, the fit preferring a slightly lower coupling constant, and the $B_{c}$ mass being better adjusted.

Tables 6 and 7 then show the same calculation but in the PS scheme. With the $m^{-1}$ potential included, the PS scheme appears to prefer lower quark masses here than the pole scheme.
Table 4 Further fits in pole scheme. Masses are in $\mathrm{MeV}$, the radiative decay width $J / \psi \rightarrow \eta_{c} \gamma$ in $\mathrm{keV}$. The infrared freezing of the running coupling constant occurs at $0.6 \mathrm{GeV}$. The recoil $1 / \mathrm{m}$ potential is not included, only the static potential

\begin{tabular}{lllll}
\hline & LO & NLO & NNLO & Expt. \\
Fit number & 1 & 2 & 3 & \\
\hline$m_{c \bar{c}}$ & 3068 & 3068 & 3068 & 3068 \\
$\Gamma_{J / \psi \rightarrow \eta_{c} \gamma}$ & 2.7 & 3.1 & 2.1 & $1.6(4)$ \\
$m_{b \bar{b}}(1 S)$ & 9458 & 9447 & 9480 & 9443 \\
$m_{b \bar{b}}(2 S)$ & 9820 & 9777 & 9769 & 10023 \\
$m_{b \bar{b}}(1 P)$ & 9899 & 9900 & 9897 & 9900 \\
$m_{b c}(1 S)$ & 5922 & 6158 & 6158 & 6317 \\
$m_{c}$ & 1720 & 1770 & 1850 & \\
$m_{b}$ & 4970 & 5040 & 5120 & \\
$\alpha_{s}$ & 0.128 & 0.116 & 0.111 & \\
\hline
\end{tabular}

Table 5 As in Table 4 but with the recoil $1 / m$ potential included

\begin{tabular}{lllll}
\hline & LO & NLO & NNLO & Expt. \\
Fit number & 4 & 5 & 6 & \\
\hline$m_{c \bar{c}}$ & 3068 & 3068 & 3068 & 3068 \\
$\Gamma_{J / \psi \rightarrow \eta_{c} \gamma}$ & 2.7 & 3.0 & 2.5 & $1.6(4)$ \\
$m_{b \bar{b}}(1 S)$ & 9443 & 9445 & 9488 & 9443 \\
$m_{b \bar{b}}(2 S)$ & 9775 & 9788 & 9772 & 10023 \\
$m_{b \bar{b}}(1 P)$ & 9900 & 9900 & 9896 & 9900 \\
$m_{b c}(1 S)$ & 6330 & 6210 & 6364 & 6317 \\
$m_{c}$ & 1740 & 1740 & 1820 & \\
$m_{b}$ & 5040 & 5000 & 5110 & \\
$\alpha_{s}$ & 0.102 & 0.106 & 0.105 & \\
\hline
\end{tabular}

Table 6 As in Table 4 but in the PS scheme with the potential totally cutoff in the infrared at $0.6 \mathrm{GeV}$

\begin{tabular}{lllll}
\hline & LO & NLO & NNLO & Expt. \\
Fit number & 7 & 8 & 9 & \\
\hline$m_{c \bar{c}}$ & 3068 & 3068 & 3068 & 3068 \\
$\Gamma_{J / \psi \rightarrow \eta_{c} \gamma}$ & 2.7 & 3.0 & 2.5 & $1.6(4)$ \\
$m_{b \bar{b}}(1 S)$ & 9462 & 9443 & 9443 & 9443 \\
$m_{b \bar{b}}(2 S)$ & 9818 & 9792 & 9789 & 10023 \\
$m_{b \bar{b}}(1 P)$ & 9898 & 9900 & 9900 & 9900 \\
$m_{b c}(1 S)$ & 5866 & 6264 & 6270 & 6317 \\
$m_{c}$ & 1740 & 1930 & 1710 & \\
$m_{b}$ & 4970 & 5230 & 5020 & \\
$\alpha_{s}$ & 0.133 & 0.112 & 0.106 & \\
\hline
\end{tabular}

Comparing Tables 8 and 9 we see that, with a lower infrared cutoff, the PS scheme does somewhat better in terms of convergence and agreement with data. 
Table 7 As in Table 4 but in the PS scheme with the potential totally cutoff in the infrared at $0.6 \mathrm{GeV}$, and with the $1 / m$ recoil potential included

\begin{tabular}{lllll}
\hline & LO & NLO & NNLO & Expt. \\
Fit number & 7 & 8 & 9 & \\
\hline$m_{c \bar{c}}$ & 3068 & 3068 & 3068 & 3068 \\
$\Gamma_{J / \psi \rightarrow \eta_{c} \gamma}$ & 2.7 & 3.0 & 2.3 & $1.6(4)$ \\
$m_{b \bar{b}}(1 S)$ & 9443 & 9443 & 9443 & 9443 \\
$m_{b \bar{b}}(2 S)$ & 9702 & 9697 & 9873 & 10023 \\
$m_{b \bar{b}}(1 P)$ & 9760 & 9772 & 10008 & 9900 \\
$m_{b c}(1 S)$ & 6070 & 6275 & 6257 & 6317 \\
$m_{c}$ & 1659 & 1634 & 1792 & \\
$m_{b}$ & 4893 & 4918 & 5091 & \\
$\alpha_{s}$ & 0.121 & 0.102 & 0.107 & \\
\hline
\end{tabular}

Table 8 Further fits in the pole scheme. Masses are in $\mathrm{MeV}$, the radiative decay width $J / \psi \rightarrow \eta_{c} \gamma$ in $\mathrm{keV}$. The infrared freezing of the running coupling constant occurs at $0.4 \mathrm{GeV}$

\begin{tabular}{lllll}
\hline Fit number & $\begin{array}{l}\text { LO } \\
1 \mathrm{a}\end{array}$ & $\begin{array}{l}\text { NLO } \\
2 \mathrm{a}\end{array}$ & $\begin{array}{l}\text { NNLO } \\
3 \mathrm{a}\end{array}$ & Expt. \\
\hline$m_{c \bar{c}}$ & 3068 & 3068 & 3068 & 3068 \\
$\Gamma_{J / \psi \rightarrow \eta_{c} \gamma}$ & 2.7 & 3.1 & 2.5 & $1.6(4)$ \\
$m_{b \bar{b}}(1 S)$ & 9542 & 9481 & 9577 & 9443 \\
$m_{b \bar{b}}(2 S)$ & 9829 & 9800 & 9804 & 10023 \\
$m_{b \bar{b}}(1 P)$ & 9891 & 9897 & 9888 & 9900 \\
$m_{b c}(1 S)$ & 6112 & 6118 & 6277 & 6317 \\
$m_{c}$ & 1667 & 1828 & 1761 & \\
$m_{b}$ & 4963 & 5117 & 5084 & \\
$\alpha_{s}$ & 0.120 & 0.121 & 0.104 & \\
\hline
\end{tabular}

Table 9 Same as Table 8 but in the PS scheme, with the potential completely cutoff in the infrared at $0.4 \mathrm{GeV}$

\begin{tabular}{lllll}
\hline & LO & NLO & NNLO & Expt. \\
Fit number & $7 \mathrm{a}$ & $8 \mathrm{a}$ & $9 \mathrm{a}$ & \\
\hline$m_{c \bar{c}}$ & 3068 & 3068 & 3068 & 3068 \\
$\Gamma_{J / \psi \rightarrow \eta_{c} \gamma}$ & 2.7 & 3.0 & 2.5 & $1.6(4)$ \\
$m_{b \bar{b}}(1 S)$ & 9614 & 9443 & 9443 & 9443 \\
$m_{b \bar{b}}(2 S)$ & 9836 & 9791 & 9788 & 10023 \\
$m_{b \bar{b}}(1 P)$ & 9884 & 9900 & 9900 & 9900 \\
$m_{b c}(1 S)$ & 6218 & 6265 & 6269 & 6317 \\
$m_{c}$ & 1631 & 1718 & 1748 & \\
$m_{b}$ & 4964 & 5029 & 5055 & \\
$\alpha_{s}$ & 0.115 & 0.112 & 0.105 & \\
\hline
\end{tabular}

Fixing the running coupling constant to either the world average or recent $\tau$ data, as in Tables 10 and 11 leads to slightly improved agreement with experiment in the NNLO
Table 10 Further fits fixing the coupling constant at the Z-pole at $\alpha_{s}\left(m_{Z}\right)=0.1204$ as determined by recent $\tau$ data. Masses are in $\mathrm{MeV}$, the radiative decay width $J / \psi \rightarrow \eta_{c} \gamma$ in $\mathrm{keV}$. The infrared freezing of the running coupling constant occurs at $0.4 \mathrm{GeV}$

\begin{tabular}{lllll}
\hline Fit number & $\begin{array}{l}\text { LO } \\
1 \mathrm{~b}\end{array}$ & $\begin{array}{l}\text { NLO } \\
2 \mathrm{~b}\end{array}$ & $\begin{array}{l}\text { NNLO } \\
3 \mathrm{~b}\end{array}$ & Expt. \\
\hline$m_{c \bar{c}}$ & 3068 & 3068 & 3068 & 3068 \\
$\Gamma_{J / \psi \rightarrow \eta_{c} \gamma}$ & 2.7 & 3.0 & 0.65 & $1.6(4)$ \\
$m_{b \bar{b}}(1 S)$ & 9444 & 9443 & 9444 & 9443 \\
$m_{b \bar{b}}(2 S)$ & 9669 & 9721 & 9586 & 10023 \\
$m_{b \bar{b}}(1 P)$ & 9718 & 9812 & 9654 & 9900 \\
$m_{b c}(1 S)$ & 6145 & 6195 & 6149 & 6317 \\
$m_{c}$ & 1629 & 1728 & 1889 & \\
$m_{b}$ & 4872 & 5000 & 5116 & \\
$\alpha_{s}$ & & & & fixed at 0.1204 \\
\hline
\end{tabular}

Table 11 Same as Table 10 but in the PS scheme, with the coupling constant cutoff at $0.4 \mathrm{GeV}$

\begin{tabular}{lllll}
\hline Fit number & $\begin{array}{l}\text { LO } \\
7 \mathrm{~b}\end{array}$ & $\begin{array}{l}\text { NLO } \\
8 \mathrm{~b}\end{array}$ & $\begin{array}{l}\text { NNLO } \\
9 \mathrm{~b}\end{array}$ & Expt. \\
\hline$m_{c \bar{c}}$ & 3068 & 3068 & 3068 & 3068 \\
$\Gamma_{J / \psi \rightarrow \eta_{c} \gamma}$ & 2.7 & 3.0 & 0.65 & $1.6(4)$ \\
$m_{b \bar{b}}(1 S)$ & 9444 & 9443 & 9443 & 9443 \\
$m_{b \bar{b}}(2 S)$ & 9646 & 9791 & 9885 & 10023 \\
$m_{b \bar{b}}(1 P)$ & 9690 & 9900 & 10034 & 9900 \\
$m_{b c}(1 S)$ & 6155 & 6264 & 6248 & 6317 \\
$m_{c}$ & 1619 & 1718 & 1918 & \\
$m_{b}$ & 4855 & 5029 & 5243 & \\
$\alpha_{s}$ & & & & fixed at 0.1204 \\
\hline
\end{tabular}

computation of the radiative transition width of the $J / \psi$, but in exchange the $1 P$ bottomonium mass is completely off.

The difference between Table 10 and entry number 9 of Table 3 is that here the global fit strategy was used while there only the $1 S$ masses were used to constrain the quark masses, and that the freezing of the coupling occurs at a slightly different momentum $(0.4$ versus $0.46 \mathrm{GeV})$.

The charm quark mass has recently [36] been reobtained from a lattice computation of the ground state charmed and charmonium mesons $\left(D, J / \psi, \eta_{c}\right)$, with $N_{f}=2$. In the $\overline{M S}$ scheme one obtains

$m_{c}^{\overline{M S}}(2 \mathrm{GeV})=1.14(4) \mathrm{GeV}$,

which translates into a mass at the charm scale of

$\bar{m}_{c}\left(\bar{m}_{c}\right)=1.28(4) \mathrm{GeV}$.

In comparing the quark mass between various schemes and scales [38], the collaboration quotes an error less than one 
standard deviation as a result of using $N_{f}=2$ instead of $N_{f}=4$.

Translating our pole-scheme masses in the various tables into $\overline{M S}$ masses $\bar{m}_{c}\left(\bar{m}_{c}\right)$ consistently yields results of order $1.3 \mathrm{GeV}$, in agreement with the lattice determination. The PS scheme on the other hand gives a somewhat larger mass of about $1.4 \mathrm{GeV}$ in the $\overline{M S}$ scheme.

To conclude this section let us quote an NLO computation with the BLM scheme, with best fit $\alpha_{s}=0.113$, $m_{c}=1750 \mathrm{MeV}$ and $m_{b}=5030 \mathrm{MeV}$. The $c \bar{c}$ radiative width is $3.0 \mathrm{keV}$. The $b \bar{b}(1 S, 2 S, 1 P)$ masses are 9393, 9775 and $9904 \mathrm{MeV}$ respectively. The $B_{c}$ mass comes out to be $6180 \mathrm{MeV}$. Thus, there is no particular advantage in using this scheme over the PS or the pole ones.

\section{Prior computations of the baryon masses}

In this section we compile existing computations of the various ground state triply heavy baryons. Since we work in the static limit, we will not resolve the hyperfine spin splitting between spin $1 / 2$ and spin $3 / 2$. Thus, in Table 12 we quote the spin average $\left(M_{1 / 2}+2 M_{3 / 2}\right) / 3$ for the $b b c$ and $c c b$ wave functions, which can appear in both spin combinations in the ground state. We further plot some of these computations in Fig. 4.

Most computations in the literature are consistent with a triply charmed baryon of around $4800 \mathrm{MeV}$, and ours will not be different. The $b b b$ baryon is preferred by most approaches in the range of $14400 \mathrm{MeV}$. A salient exception is the sum-rule computation of [39] that seems to be significantly lower.

Closest in spirit to our approach is the Coulombic calculation of Jia [40], which could be considered a Leading
Order pNRQCD computation of the static potential. Indeed, this author employs Eq. (21), with parameters specified in Table 13. The error bar quoted in that work corresponds to the author's estimate of higher orders in perturbation theory. We will explore the systematics of this computation, extending it in several ways. First, we will work to two higher orders in perturbation theory with the potentials now available. Thus, we will ascertain that this error was underestimated. Second, we will quantify the error implicit in the one-wave function variational approximation (that Jia also uses) by showing explicit computations in very similar atomic systems for which the experimental data are available. Third, we will incorporate a running coupling constant at all steps, and handle the attending infrared systematic uncertainties by comparing different methods. The outcome of our work will thus be a much more detailed understanding of triply heavy baryons in the context of pNRQCD.

It is also worth remarking that in [41] the difference between the triply heavy baryon mass with a $\Delta$-like two-body potential and an $\Upsilon$ type potential has been reported in a variational model computation. We have plotted their results in Fig. 5. As is easily seen, those authors find that the $\Upsilon$ configuration is slightly heavier, but in any case the difference is only of order $20-40 \mathrm{MeV}$.

Table 13 Parameters employed by Yu Jia in an early variational computation of the triply heavy baryon spectrum with a strong Coulomb potential. The coupling constant is given at the charm scale (employed in the $c c c$ computation) and the bottom soft scale (employed for all others)

\begin{tabular}{ll}
\hline$\alpha_{s}(0.9 \mathrm{GeV})$ & 0.59 \\
$\alpha_{s}(1.2 \mathrm{GeV})$ & 0.43 \\
$m_{c}=\frac{M_{J / \psi}}{2}\left(1+2 \frac{2 \alpha_{s}^{2}}{9}\right)$ & $\simeq 1.668 \mathrm{GeV}$ \\
$m_{b}=\frac{M_{r}}{2}\left(1+2 \frac{2 \alpha_{s}^{2}}{9}\right)$ & $\simeq 4.924 \mathrm{GeV}$ \\
\hline
\end{tabular}

Table 12 Computations of triply heavy baryon masses. Many of the entries were already compared in [41] but we have added more information available in the literature

\begin{tabular}{llllll}
\hline Method & Ref. & $M_{c c c}$ & $M_{c c b}$ & $M_{b b c}$ & $M_{b b b}$ \\
\hline Variational Coulomb & {$[40]$} & $4760(60)$ & $7980(70)$ & $11190(80)$ & $14370(80)$ \\
Variational Cornell & {$[41]$} & 4799 & 8037 & 11235 & 14398 \\
Faddeev & {$[42]$} & 4799 & 8019 & 11217 & 14398 \\
Bag model & {$[43]$} & 4790 & 8030 & 11200 & 14300 \\
Quark counting rules & {$[47]$} & $4925(90)$ & $8200(90)$ & $11480(120)$ & $14760(180)$ \\
Const. quark model & {$[48]$} & 4965 & 8258 & 11548 & 14834 \\
Const. quark model & {$[49]$} & 4632 & & & \\
Relat. quark model & {$[50]$} & 4803 & 8023 & 11285 & 14569 \\
Instanton quark model & {$[51]$} & 4773 & & & 14451 \\
Hypercentral model & {$[52]$} & 4736 & 8096 & 11381 & $13280(100)$ \\
Sum rules & {$[39]$} & $4670(150)$ & $7443(150)$ & $10460(110)$ & $14371(12)$ \\
Lattice & {$[53]$} & 4780 & & & $14700(300)$ \\
Regge estimate & {$[54]$} & $4819(7)$ & & & $11400(300)$ \\
pNRQCD & This work & $4900(250)$ & $8150(300)$ & & \\
\hline
\end{tabular}



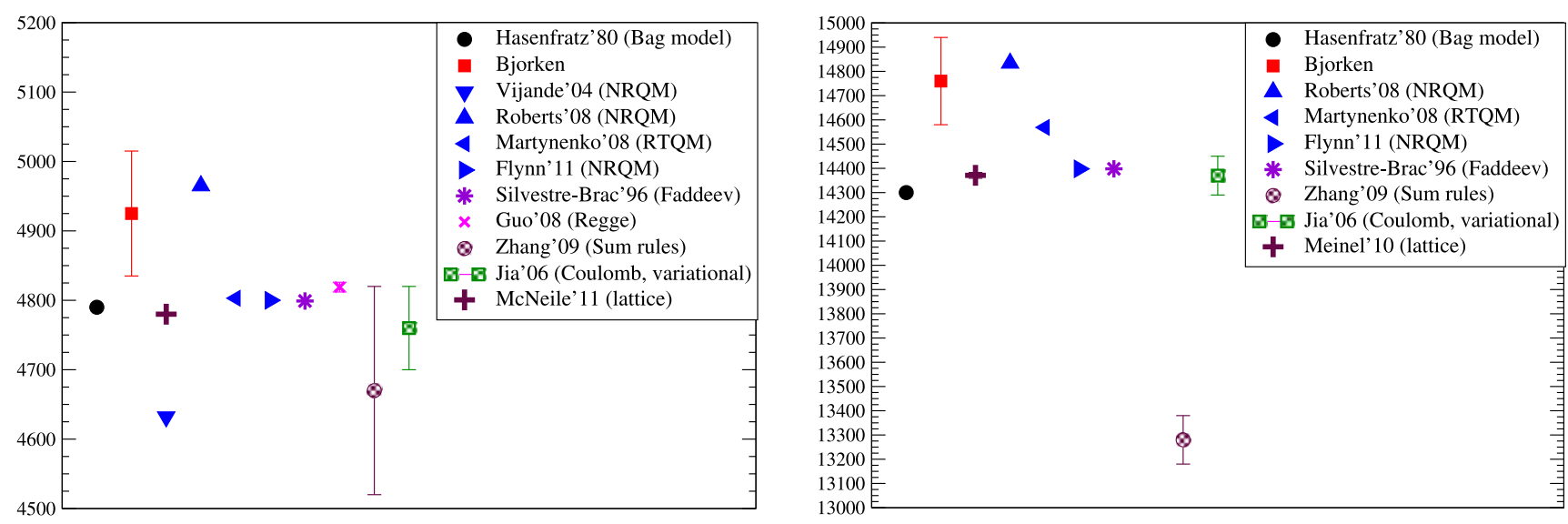

Fig. 4 Scatter of several existing computations for the $\Omega_{c c c}$ and $\Omega_{b b b}$ masses, respectively. See Table 12 for references

Fig. 5 Mass difference between triply heavy baryons computed with the $\Delta$ type potential (two-body interactions alone) and $\Upsilon$-type potential (three-body interactions alone) reported by Flynn, Hernández and Nieves in [41]
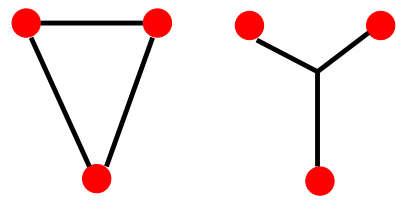

This observation is of interest for light-quark baryons, since they can be more conveniently treated by covariant means, but the (much simplified) Faddeev equations require a vanishing pure three-body kernel. That there is not much difference between $\Delta$ and $\Upsilon$ shape configurations is important information for establishing that the Faddeev equations are approximately valid, at least in the heavy quark limit when the soft scale is perturbative. ${ }^{4}$

In our perturbative treatment for heavy baryons we will compare the mass computation with and without the pure three-body force that appears at NNLO in perturbation theory, finding that this difference is also small, and thus further reinforcing the conclusion of Flynn et al.

\section{Novel computation of triply heavy baryon masses}

We treat the three-body problem in a similar manner to [57, 58] variationally by employing a simple wave-function

\footnotetext{
${ }^{4}$ Should the soft-scale be non-perturbative, one could obtain some information from other published work $[55,56]$, but incorporating this in a three-body computation is currently beyond our scope.
}

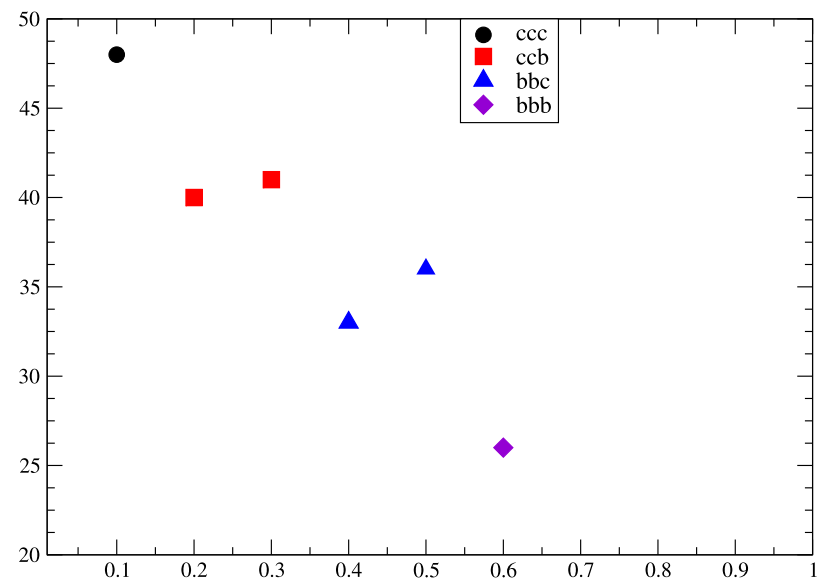

ansatz and computing the expectation value of the pNRQCD Hamiltonian. The Rayleigh-Ritz variational principle guarantees that the outcome is an upper bound of the true ground state energy in the given channel,

$\frac{\left\langle\psi_{\alpha_{\rho} \alpha_{\lambda}}\left|H_{\mathrm{pNRQCD}}\right| \psi_{\alpha_{\rho} \alpha_{\lambda}}\right\rangle}{\left\langle\psi_{\alpha_{\rho} \alpha_{\lambda}} \mid \psi_{\alpha_{\rho} \alpha_{\lambda}}\right\rangle} \geq E_{0}$.

The two parameters $\alpha_{\rho}, \alpha_{\lambda}$ are then varied to find the best possible upper bound on energy for the given ansatz. These two parameters are associated to two momentum-space Jacobi coordinate vectors, the third independent vector being fixed by the center of mass condition (hadron at rest):

$k_{\rho}=\frac{k_{1}-k_{2}}{\sqrt{2}}$

$k_{\lambda}=\sqrt{\frac{3}{2}}\left(k_{1}+k_{2}\right)$,

$k_{3}=-k_{1}-k_{2}$.

We choose as ansatz

$\psi\left(k_{\rho}, k_{\lambda}\right)_{\alpha_{\rho} \alpha_{\lambda}}=Y_{00}\left(k_{\rho}\right) Y_{00}\left(k_{\lambda}\right) e^{-k_{\rho} / \alpha_{\rho}-k_{\lambda} / \alpha_{\lambda}}$, 
which gives reasonable results (we have also checked other forms such as a rational function). The error incurred in this variational approximation is estimated below in Sect. 9. The wave function in Eq. (40) is symmetrized as needed by invoking it in the computer programme with different arguments, to guarantee symmetry under exchange of any two equal quarks. The color singlet wave function $\epsilon_{i j k} / \sqrt{3}$, which is implicit in the calculation (and has already been used in the computation of the color factors of the various potentials), is then responsible for the antisymmetry expected under fermion exchange.

In practice we compute the Hamiltonian's expectation value for the three-body problem in momentum space.

\subsection{Results in the PS scheme}

With the three parameters $\alpha_{s}, m_{c}, m_{b}$ in the PS scheme fit to the meson spectrum, the only sensitivity left to explore is that of the infrared cutoff scale. In Tables 14 and 15 we present the outcome of the three-body computation in the PS scheme.

The mass values obtained are significantly higher than in other approaches. As will be seen in the next section, this is a feature of the PS scheme that misses quite some of the binding, as opposed to the pole scheme. This feeling is reinforced by the observation that the computation with the lower $0.4 \mathrm{GeV}$ cutoff does much better, both in terms of binding and convergence. Particularly bad is the computation with an infrared cutoff at $0.6 \mathrm{GeV}$ at NLO, which yields an unbelievably high mass.

The results in this and the next subsection satisfy Nussinov's inequalities [44, 45]. The first,

$M_{\Omega_{b b c}} \leq 2 M_{\Omega_{c c b}}-M_{\Omega_{c c c}}$

Table 14 Ground state triply heavy baryon masses in the PS scheme, with infrared cutoff $\lambda=0.4 \mathrm{GeV}$, for various orders of perturbation theory. All masses in $\mathrm{MeV}$

\begin{tabular}{lllll}
\hline Order & $c c c$ & $c c b$ & $b b c$ & $b b b$ \\
\hline LO & 4895 & 8235 & 11535 & 14770 \\
NLO & 5160 & 8480 & 11750 & 14970 \\
NNLO & 5250 & 8560 & 11805 & 15040 \\
\hline
\end{tabular}

Table 15 Ground state triply heavy baryon masses in the PS scheme, with infrared cutoff $\lambda=0.6 \mathrm{GeV}$, for various orders of perturbation theory. All masses in $\mathrm{MeV}$

\begin{tabular}{lllll}
\hline Order & $c c c$ & $c c b$ & $b b c$ & $b b b$ \\
\hline LO & 5240 & 8500 & 11640 & 14750 \\
NLO & 5810 & 9170 & 12460 & 15670 \\
NNLO & 5150 & 8690 & 12100 & 15500 \\
\hline
\end{tabular}

is a consequence of heavier systems being more bound than lighter systems (as discussed at the end of Sect. 2.1). The second inequality, satisfied by a sizeable amount, reads

$M_{\Omega_{b b c}} \geq \frac{M_{\Upsilon}}{2}+M_{B_{c}}$

and means that mesons are more tightly bound than baryons. They are well satisfied when the three-body computation is compared to the corresponding two-body computation under the same scheme and conditions employed for parameter fitting.

\subsection{Results in the pole scheme}

Tables 16 and 18 present our results in the pole scheme with coupling freezing at 0.6 and $0.4 \mathrm{GeV}$ respectively.

Comparing Tables 16 and 14 we see that the PS scheme, with its drastic infrared cutoff to avoid renormalons, is underestimating the binding energy by a large amount of order $300 \mathrm{MeV}$. To check whether this is ameliorated for yet heavier quarks we have ran also with $m_{Q}=10 \mathrm{GeV}$, a quark twice as heavy as the bottom, and with $m_{Q}=15 \mathrm{GeV}$. The results are shown in Table 17.

For the $10 \mathrm{GeV}$ quark we obtain a mass of $29.22 \mathrm{GeV}$ in the pole and $29.67 \mathrm{GeV}$ in the PS scheme. Although the difference is now a smaller percentage of the total mass, it is still very significant in absolute terms. Therefore we do not expect that a small refitting of parameters, such as quark masses or coupling constant, will be able to eliminate it within the present setup.

Table 16 Ground state triply heavy baryon masses in the pole scheme, with infrared freezing point $\lambda=0.4 \mathrm{GeV}$, for various orders of perturbation theory. All masses in $\mathrm{MeV}$

\begin{tabular}{lllll}
\hline Order & $c c c$ & $c c b$ & $b b c$ & $b b b$ \\
\hline LO & 4708 & 7975 & 11180 & 14386 \\
NLO & 4900 & 8140 & 10890 & 14500 \\
NNLO & 4865 & 8150 & 11400 & 14683 \\
\hline
\end{tabular}

Table 17 The difference between the PS scheme and the pole scheme seems to persist at masses twice and thrice as big as the bottom quark, with the PS scheme underestimating the binding energy. Although for asymptotically large masses we believe that this difference should ameliorate, we do not see it presently

\begin{tabular}{lll}
\hline Scheme & Quark mass $(\mathrm{GeV})$ & Baryon mass $(\mathrm{GeV})$ \\
\hline Pole & 5.08 & 14.68 \\
PS & 5.06 & 15.04 \\
Pole & 10 & 29.22 \\
PS & 10 & 29.67 \\
Pole & 15 & 43.95 \\
PS & 15 & 44.46 \\
\hline
\end{tabular}


After all numbers have been considered, we deem that the computation that has the best balance between convergence of perturbation theory and capture of the infrared physics is that in Table 16. To obtain the best estimate of the physical baryon mass, the results computed there have to be extrapolated by increasing the binding energy by $25 \%$ to compensate for the variational approximation (see Sect. 9).

Figure 6 compares the results in the PS and pole schemes with an infrared saturation scale of $0.6 \mathrm{GeV}$, to the three orders of perturbation theory available.

Several conclusions follow from the figure. First, it is plain that Jia's calculation is in the right ballpark, but underestimates the corrections due to higher orders of perturbation theory (note that our coupling constant is on the low side of the world average, such that a scheme that will reduce these corrections is hard to imagine). In addition, one sees that as already mentioned, the PS scheme probably underestimates the binding in our current treatment. Finally, and taking into account the variational error bar (any such calculation also underestimates the binding), the prediction for the $c c c$ mass should be about $4800 \mathrm{MeV}$. We later will correct this figure up when accounting for the $V_{m^{-1}}$ potential in Sect. 8.4.

Table 18 Ground state triply heavy baryon masses in the pole scheme, with infrared freezing point $\lambda=0.6 \mathrm{GeV}$, for various orders of perturbation theory. All masses in $\mathrm{MeV}$

\begin{tabular}{lllll}
\hline Order & $c c c$ & $c c b$ & $b b c$ & $b b b$ \\
\hline LO & 4750 & 7950 & 11100 & 14200 \\
NLO & 5050 & 8290 & 11470 & 14630 \\
NNLO & 4970 & 8200 & 11340 & 14570 \\
\hline
\end{tabular}

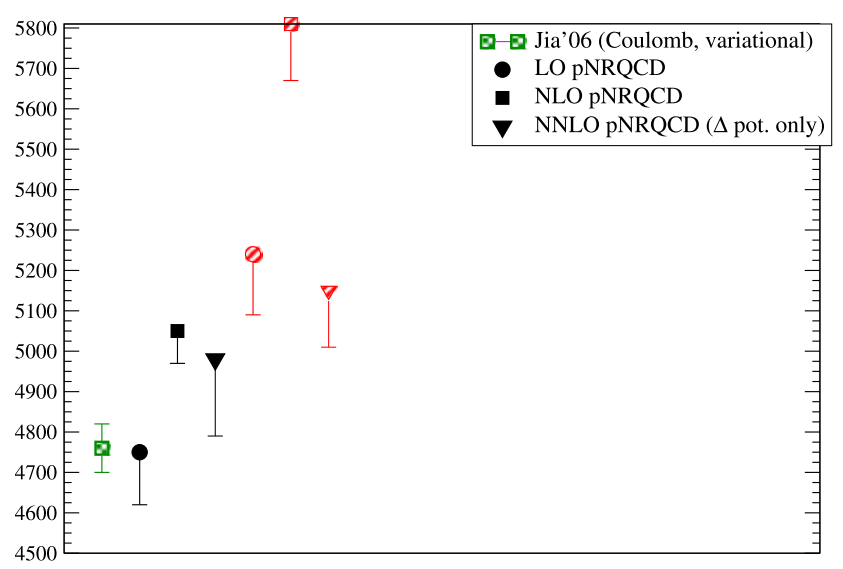

Fig. $6 \Omega_{c c c}$ computations in pole scheme (solid) and PS scheme (shaded, red online) to LO, NLO and NNLO. The leftmost point (green online) is the original Coulomb evaluation by Yu Jia with his quoted error band estimating the NLO effect. Infrared saturation (in the pole scheme) or cutoff (in the PS scheme) set at $0.6 \mathrm{GeV}$. The asymmetric error band is our extrapolation of the missing binding energy due to the variational wave function

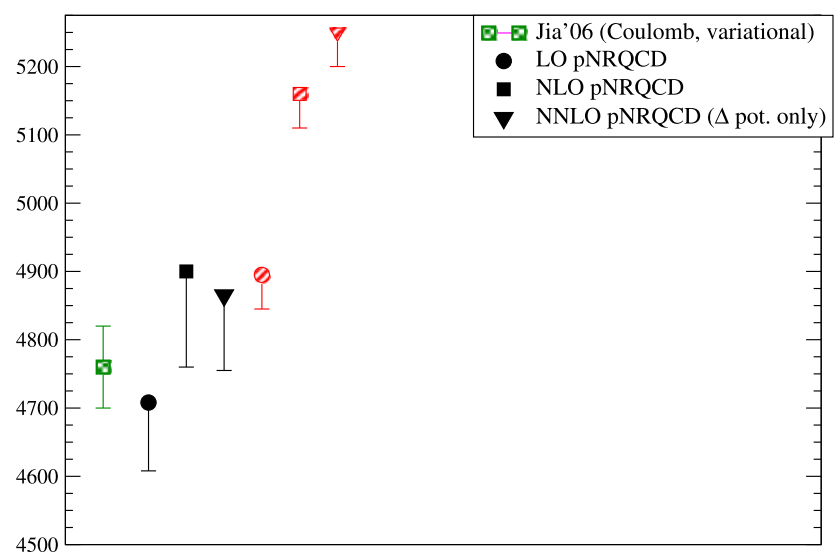

Fig. 7 Same as in Fig. 6 but for the IR saturation/cutoff at a lower scale of $0.4 \mathrm{GeV}$

Comparing with the results plotted in Fig. 7, we see that the region between 0.6 and $0.4 \mathrm{GeV}$ still contributes at least an additional $100 \mathrm{MeV}$ of binding.

Figure 8 then plots the predictions for the other (spinaveraged) triply heavy baryons (in the pole scheme) and gives a panoramic of other results in the literature.

Figure 9 shows the size of the binding energy from the three-body variational calculation by comparing it with three times the pole mass (given that the potential we use is extracted from perturbation theory, this acts as a dissociation threshold, which should not be present in a lattice or a Cornell model computation, for example). It is plain that, although separately the pole mass and the binding energy do not converge well, there is a cancelation between them that helps the behavior of the baryon mass in perturbation theory.

\subsection{Effect of the three-body force}

Next we address the difference between a computation employing the intrinsic three-body force, and a computation with only the two-body force. If we set the scale in Eq. (26) according to the "hard scale" prescription

$\alpha_{s}^{3} \rightarrow \alpha_{s}\left(m_{c}\right)^{3}$

we obtain a very small pure three-body contribution of order $1 \mathrm{MeV}$. If instead, in view of the typical momentum transfer through the three gluons, we choose the more sensible "soft scale" prescription

$\alpha_{s}^{3} \rightarrow \alpha_{s}\left(q_{2}\right) \alpha_{s}\left(q_{3}\right) \alpha_{s}\left(\sqrt{q_{2} q_{3}}\right)$

the effect of the (perturbative) three-body force is of order $20-40 \mathrm{MeV}$, in broad agreement with the related (though not equivalent) estimates of Flynn et al. The result of the computation of the three-body potential for the different flavor combinations is depicted in Fig. 10. The effect we find is of 


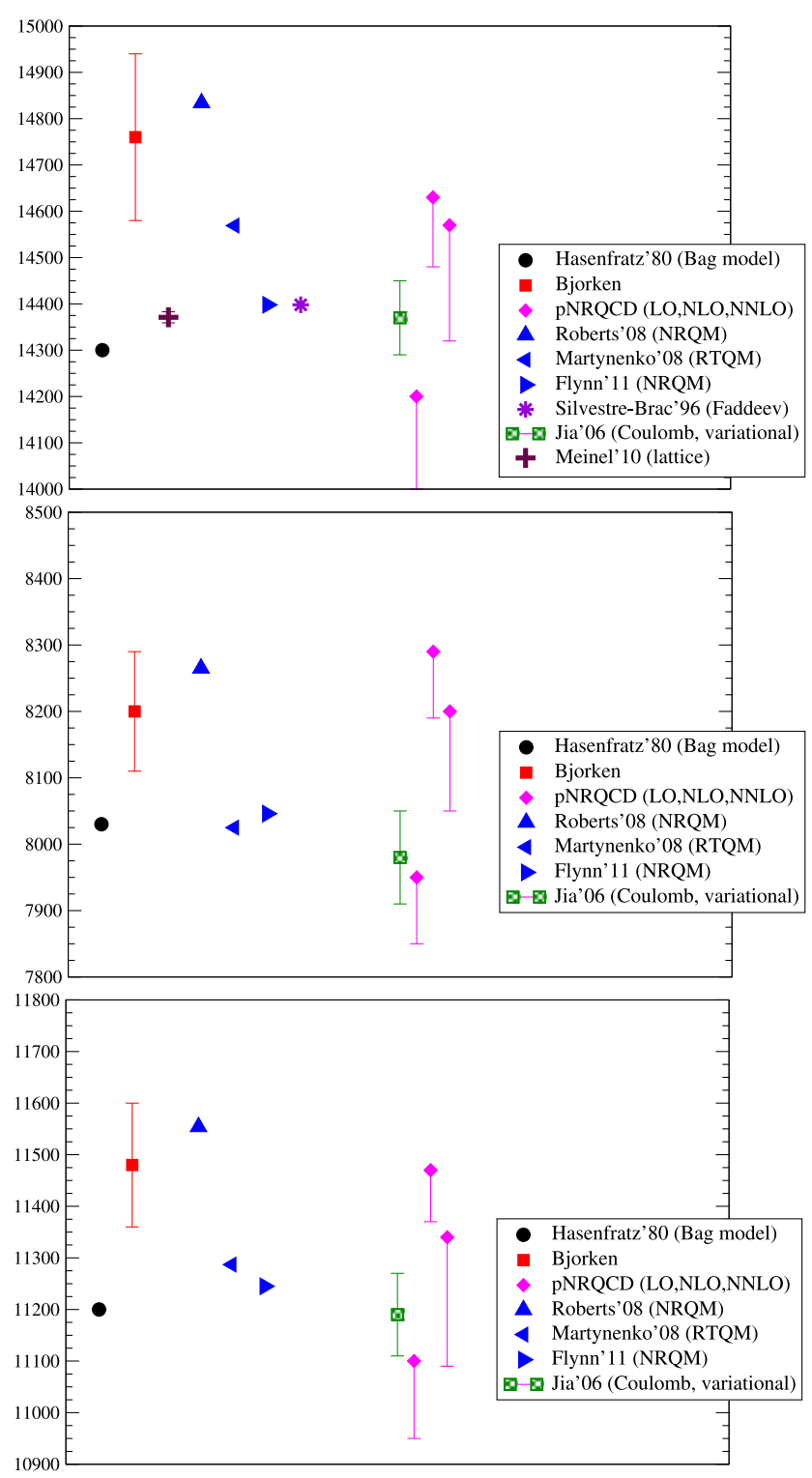

Fig. 8 Predictions for the mass of the $\Omega_{b b b}$, and spin averaged $c c b$, $b b c$ combining results analogous to those of Fig. 6 and including other computations as in Fig. 4

size $17 \mathrm{MeV}$ for $c c c, 25 \mathrm{MeV}$ for $c c b, 39 \mathrm{MeV}$ for $b b c$ and $20 \mathrm{MeV}$ for $b b b$, with an error of about $5 \mathrm{MeV}$ or less.

The immediate conclusion is that in the heavy quark limit, intrinsic three-body forces (defined as those vanishing when one quark is put far away from the other two) are small in ground state baryons.

\subsection{Effect of the $1 / m$ potential}

Thus far our three-quark results have been based on the purely static potential. In this section we lift this approximation and study, at NLO, the effect of adding a $V_{m^{-1}}$ contribution. This recoil correction has not been worked out in detail in the literature, so we abstain from attempting an NNLO

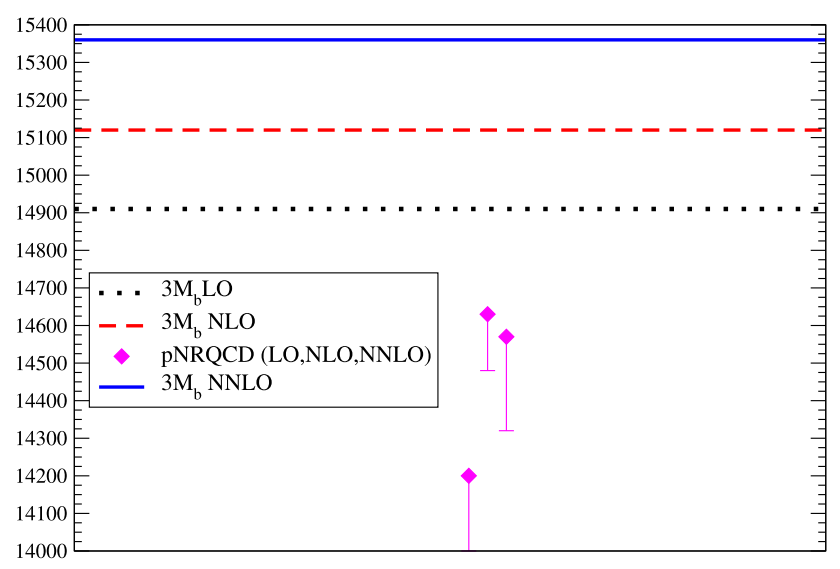

Fig. 9 Binding energy of the triple- $b$ system. Lines: three times the pole mass (from bottom to top LO, NLO, NNLO). Symbols: mass of the $\Omega_{b b b}$. The graph shows how the resonance mass is better behaved in perturbation theory than either of the pole mass or the binding energy

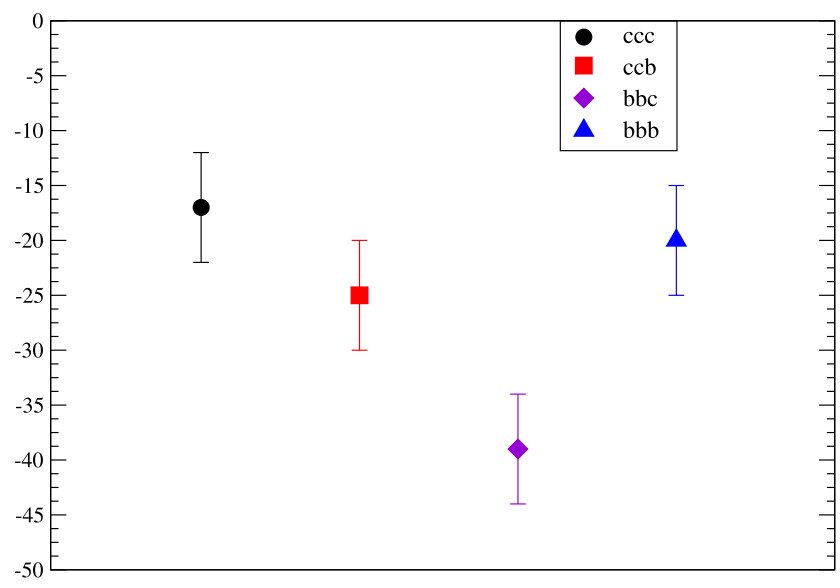

Fig. 10 The effect of adding the three-body force to the NNLO potential is to increase the binding in the amounts visible. The effect seems smaller for equal flavor objects than for mixed flavor, and does not depend much on quark masses

Fig. 11 Non-Abelian diagram that produces a recoil $V_{m^{-1}}$ potential in triply heavy baryons at NLO

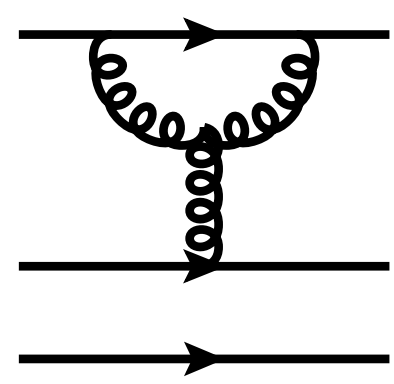

evaluation. But if we turn to the simplest convention of [7], the NLO $V_{m^{-1}}$ is entirely given by the non-Abelian diagram with a three gluon vertex, whose equivalent for baryons is sketched in Fig. 11. 


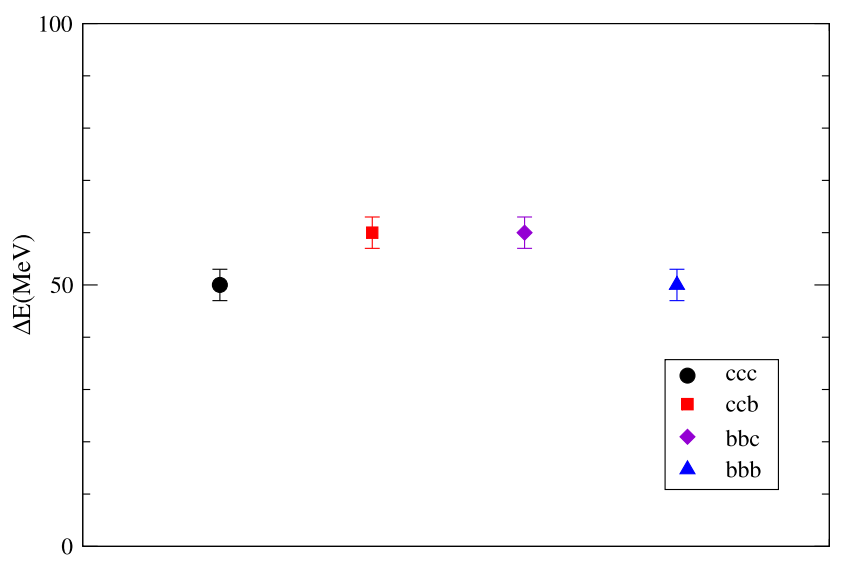

Fig. 12 Effect of $V_{m^{-1}}$ at NLO from Eq. (13) on the ground-state baryon spectrum. Plotted is $M_{1}-M_{0}$, the mass difference including the $1 / m$ potential or employing the static potential alone. The mass of the $\Omega_{c c c}$ can be raised by some $50 \mathrm{MeV}$ due to recoil corrections

Because the interaction is two-body, the potential in Eq. (13) can immediately be adopted for baryons, with appropriate kinematics and excepting a color factor.

The latter can be worked out easily by noting that the diagram is a one-loop radiative correction to the quark-gluon vertex on the quark at the very top of Fig. 11. This nonAbelian vertex correction is easily seen to increase the usual Gell-Mann color matrix at the vertex [46]

$T^{a} \rightarrow \frac{N_{c}}{2} T^{a}$

Thus the ratio of color factor in baryons over color factor in mesons is the same for the static potential as for the recoil correction, to NLO and in the convention of [7]

$\frac{C_{3, m^{-1}}}{C_{2, m^{-1}}}=\frac{C_{3,0}}{C_{2,0}}$

and in practice it is sufficient to divide Eq. (13) by a factor 2 to obtain each of the three possible two-body interactions in the baryon system.

Next we will show the difference in baryon mass with and without the $V_{m^{-1}}$ potential, consistent with Table 7. To be consistent with the given order in perturbation theory, the coupling constant runs only at NLO.

The difference between computing baryon masses with the recoil potential or without it at NLO is depicted in Fig. 12. As can be seen, the effect is quite independent of quark masses from $c c c$ to $b b b$ as discussed earlier around Eq. (16).

\section{Error estimates}

All integrals in the computation of the three-body Hamiltonian matrix element are evaluated by Monte-Carlo methods and we allow an error of $10 \mathrm{MeV}$ in their computation, except in our three-body force or recoil force computations. In those we have demanded an error in the $1-5 \mathrm{MeV}$ range given that we have to subtract two masses. This numerical uncertainty will be negligible in the final error balance.

To estimate the variational errors we turn to some simple systems in atomic and molecular physics that can be addressed with the same techniques, providing in addition a check of the computer programmes. We take three-body systems made of one electron and two protons (the dihydrogen cation $\mathrm{H}_{2}^{+}$), and one $\alpha$-particle binding two electrons with parallel or antiparallel spins (ortho- and para-helium, respectively). These are depicted in Fig. 13.

Although we are not considering spin interactions, the distinction between ortho- and para-helium is also important as it checks our wave-function symmetrization procedure.

We give the matrix elements in terms of reduced momenta $\tilde{k} \equiv k /\left(m_{e} \alpha_{e m}\right)$. They read, for atomic helium,

$$
\begin{aligned}
\langle H\rangle_{\psi}= & m_{e} \alpha_{e m}^{2} \int \frac{d^{3} k_{1}}{(2 \pi)^{3}} \frac{d^{3} k_{2}}{(2 \pi)^{3}} \psi^{*}\left(k_{1}, k_{2}\right) \\
& \times\left[\frac{1}{2}\left(\tilde{k}_{1}^{2}+\tilde{k}_{2}^{2}+\tilde{k}_{3}^{2} \frac{m_{e}}{m_{\alpha}}\right) \psi\left(k_{1}, k_{2}\right)\right. \\
& +\int \frac{4 \pi d^{3} q}{(2 \pi)^{3} q^{2}}\left(\psi\left(k_{1}+q, k_{2}-q\right)\right. \\
& \left.\left.-2 \psi\left(k_{1}+q, k_{2}\right)-2 \psi\left(k_{1}, k_{2}+q\right)\right)\right]
\end{aligned}
$$

and for the dihydrogen cation,

$$
\begin{aligned}
\langle H\rangle_{\psi}= & m_{e} \alpha_{e m}^{2} \int \frac{d^{3} k_{1}}{(2 \pi)^{3}} \frac{d^{3} k_{2}}{(2 \pi)^{3}} \psi^{*}\left(k_{1}, k_{2}\right) \\
& \times\left[\frac{1}{2}\left(\tilde{k}_{1}^{2}+\left(\tilde{k}_{2}^{2}+\tilde{k}_{3}^{2}\right) \frac{m_{e}}{m_{p}}\right) \psi\left(k_{1}, k_{2}\right)\right. \\
& +\int \frac{4 \pi d^{3} q}{(2 \pi)^{3} q^{2}}\left(-\psi\left(k_{1}+q, k_{2}-q\right)\right. \\
& \left.\left.-\psi\left(k_{1}+q, k_{2}\right)+\psi\left(k_{1}, k_{2}+q\right)\right)\right]
\end{aligned}
$$

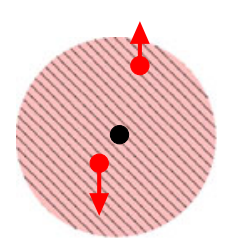

Para-Helium

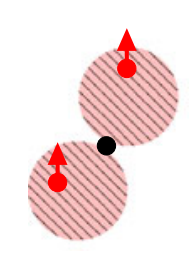

Ortho-Helium

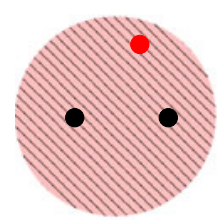

Dihydrogen cation
Fig. 13 Three-body systems in atomic physics that we use to test the variational method and computer programme. From left to right, para-helium (one $\alpha$ particle and two electrons with spin antialigned), ortho-helium (the two electron spins are now aligned) and the hydrogen cation (two protons loosely bound by one electron alone) 
where the $4 \pi / q^{2}$ Coulomb potential is clearly recognizable, and makes the expression very much alike to our heavybaryon computation at LO.

The outcome of these atomic computations is then plotted in Fig. 14.

From this exercise we estimate the precision of the simple one-wave function, two-parameter variational estimate, to be about $25 \%$ in error in the computation of the binding energy. This error can eventually be reduced to zero by a systematic shell by shell diagonalization with a large basis, and we are planning such undertaking, but it exceeds the purposes of the present work.

For now we just note that since the Rayleigh-Ritz variational principle guarantees that the computed state is less bound than the physical state, we can reduce the error by the device of increasing the binding energy that estimated $25 \%$ in the final quoted estimate. This extrapolation is assisted by our atomic physics computation, since once the respective scales are pulled out of the matrix elements for heavy baryons or for atomic helium, they are very similar.

Returning next to heavy baryons, we observe that perturbation theory seems to be converging reasonably well, and that, while the jump from LO to NLO is appreciable, the difference between NLO and NNLO is substantially smaller and of order $100 \mathrm{MeV}$ at most. This reasoning applies also to higher order recoil corrections.

We also incur in a small inaccuracy of order 5-10 MeV in the computation of the $c c b$ and $b b c$ mixed-flavor mesons in employing $N_{f}=3$ in the appropriate coupling constant,

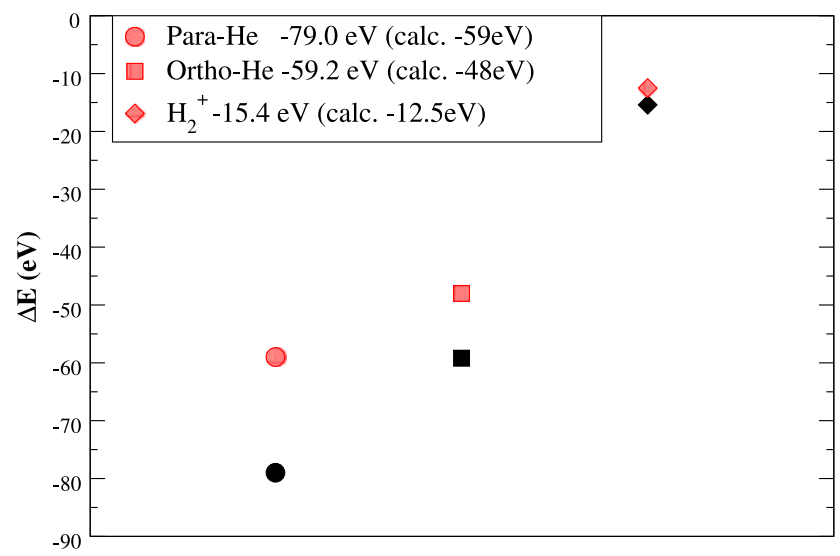

Fig. 14 Variational estimate of the various binding energies in atomic three-body problems, together with the experimental values instead of varying the screening $N_{f}$ with the scale of the various interactions, which may require $N_{f}=3$ or $N_{f}=4$. This is in order to simplify and speed the execution of computer programmes. In neglecting the charm sea in these baryons we are in line with many other modern computations. $N_{f}=4$ is correctly set for $b b b$ baryons.

The error is therefore dominated by the treatment of the infrared. The Potential Subtracted Scheme offers perhaps slightly improved convergence in perturbation theory, but since the potential is completely truncated at a low scale, it underestimates the binding energy systematically, and therefore overestimates the mass by a significant amount (several hundred $\mathrm{MeV}$ ).

This notorious effect should be absent for infinitely heavy quarks, where $m v \gg \Lambda_{\mathrm{QCD}}$, but since for physical charm and bottom quarks the scale separation is not very clean, we see that imposing an infrared cutoff as the PS scheme demands affects the computed binding energy.

Turning to the pole scheme with saturated running constant in the infrared, we see that the binding energy is more in line with other approaches, and the convergence of perturbation theory is still acceptable. However the difference between the two approaches advises us to assign an error of order $200 \mathrm{MeV}$ to the result.

That this infrared sensitivity is caused by the finiteness of the quark mass can be exposed by the following reasoning. In the case of the hydrogen atom, the binding caused by momenta smaller than the inverse Bohr radius $1 / a_{0}$ is about $13 \%$ of the total $-13.6 \mathrm{eV}$. In QCD the running coupling constant continues growing below $a_{0}^{-1}$ which is of order $500 \mathrm{MeV}$, causing a correspondingly higher error. For quarks of much larger mass however, $a_{0}^{-1}$ is higher and the low momentum tail of the wave function has little overlap with the infrared region where $\Lambda_{\mathrm{QCD}}$ influences the result.

To summarize this section, our estimate of the reasonable uncertainties for the $\Omega_{c c c}$ is given in Table 19 .

\section{Prospects for experimental detection}

James Bjorken [47] proposed several decay chains accessible to experiment that would allow the reconstruction of the $\Omega_{c c c}$. More recently, Chen and $\mathrm{Wu}$ [59] have estimated that $10 \mathrm{fb}^{-1}$ of LHC integrated luminosity recorded by a detector would contain $10^{4}$ to $10^{5}$ triply charmed baryons. They
Table 19 Estimate of various uncertainties that affect our computation of the $\Omega_{c c c}$ mass. The current error is clearly dominated by the treatment of the infrared. A factor of 2 in error reduction could be gained in future, more sophisticated work. The various errors are from uncorrelated sources. The error for other flavor combinations is easily $50-100 \mathrm{MeV}$ larger at this stage of the analysis

\begin{tabular}{lllllll}
\hline Source & Parameters & Monte Carlo & Pert. theory & IR cutoff & Variational & Total \\
Uncertainty $(\mathrm{MeV})$ & 50 & 10 & 100 & 200 & 50 & 250 \\
\hline
\end{tabular}


further propose looking for the particular decay chain

$\Omega_{c c c} \rightarrow \Omega_{s s s} \pi \pi \pi$

We agree that this channel provides in principle a very clean signature, since all four final state particles are charged and can be identified by $d E / d x$ energy deposition on a tracking chamber. However, the tremendous combinatorial background that the LHC experiments have to contend with, often with hundreds of pions in a single event, make the search extremely difficult.

The reconstruction could be performed also at other experiments, such as perhaps COMPASS [61, 62] and certainly the $B$ factories, operating at less energy and thus with less multi-particle production. These, particularly COMPASS, are however limited in statistics and produce less triple charm events. Therefore, it would be an advantage to combine the spectra in all Cabibbo-allowed four charged particle channels $\Omega_{s s s} \pi \pi \pi, \Xi K \pi \pi, \Sigma K K \pi$, and $p K K K$.

Still, an alternative route to complete reconstruction of the $\Omega_{c c c}$ would be to at least measure its mass in a recoil spectrum. Since charm is produced by the strong interactions in $c \bar{c}$ pairs, the $\Omega_{c c c}$ needs to recoil against three charm antiquarks, most often in the form of three $\bar{D}$ mesons, for example in the reaction

$e^{-} e^{+} \rightarrow \Omega_{c c c} \bar{p} \overline{D D D}$,

which, depending on the energy, can be accompanied by any number of pions.

The interesting feature to exploit is that the $\Omega_{c c c}$ is the lightest state that can carry three charm quarks. Thus, an alternative strategy to reconstructing a decay chain, any of which will have small branching fractions, would be to search for the recoiling triple (anti)charm system and recoil antiproton.

Since no triple charm spectrum has ever been published to our knowledge, searching for three $\bar{D}$ mesons is an interesting undertaking in itself. This can be accomplished, for example, by a lepton trigger that tags one of the charm mesons (only $50 \%$ inefficient while suppressing much background), followed by reconstruction of the other two, or by a pure hadronic trigger in which all three are fully reconstructed.

The further identification of a recoil antiproton in a small subset of the events immediately provides an upper bound on the $\Omega_{c c c}$ mass, simply by the missing energy technique against the recoiling system, even if the $\Omega_{c c c}$ itself was not fully reconstructed.

Baranov and Slad [60] estimated that the production cross section for $\Omega_{c c c} \overline{D D D}$ at the $Z$-pole in $e^{-} e^{+}$collisions is of order $0.04 \mathrm{fb}^{-1}$, which is too small to have been usable at LEP or SLC. However, we should take into account that the $\frac{1}{s}$ flux factor allows for a larger cross-section, up to perhaps $3 \mathrm{fb}$, in the $10 \mathrm{GeV}$ region where the $B$ factories operate. Such cross-section is not unreasonable taking into account that the B-factories have measured double charm and charmonium cross-sections. For example, for the very exclusive channel $e^{+} e^{-} \rightarrow J / \psi+\eta_{c}$, Belle finds a cross section of about 26(6) femtobarn, while Babar reports some 18(5) femtobarn. Exclusive triple charmonium channels should be another two to three orders of magnitude smaller, but open flavor channels as the one we propose in Eq. (46) will be affected less by wave-function suppression, if the accelerator reaches sufficient energy.

Adding the masses of all the particles, we find a threshold

$M_{\Omega_{c c c}}+M_{p}+3 M_{\bar{D}} \simeq 11440(250) \mathrm{MeV}$

which lies at a slightly higher energy than Belle's data base at the $\Upsilon(5 S)$, taken around $10860 \mathrm{MeV}$.

It should be feasible for Super-B and Belle-II to take data at slightly higher energies around the $\Upsilon(6 S)$ resonance and try to identify a triply charmed spectrum.

As for the detection of an effect of the three-body force in the ground state spectrum, we do not share the optimism in Ref. [41], since the $25 \mathrm{MeV}$ effect has to be found by comparing experimental data with detailed theoretical predictions of the masses, which, as we have shown, have systematic errors larger by an order of magnitude. A cleaner way of extracting this information will have to be devised.

\section{Outlook}

We believe that we have presented a stride beyond the computation of Jia for triply heavy baryons. In this initial evaluation we have employed the newly available NLO and NNLO potentials, obtaining results broadly consistent with other approaches, for the static potential, and also with recoil corrections. Our prediction for the $\Omega_{c c c}$ mass is 4900 (250) MeV. For the ccb, bbc, and bbb combinations we respectively predict 8150,11400 and $14700 \mathrm{MeV}$ with a temptative error of $300 \mathrm{MeV}$.

Although at this point our prediction of the $\Omega_{c c c}$ mass is not particularly precise, it is information stemming from pNRQCD, the appropriate effective theory of QCD for ground state triply heavy baryons, a qualitative improvement over the present, largely model-based situation. Computations by lattice groups are also underway.

In future work we intend to reduce the uncertainties in this work by employing an additional, more sophisticated R-evolution [68] scheme that implements a renormalization group philosophy to deal with the infrared cutoff dependence, and by attempting a multi-wave function systematic diagonalization. These two undertakings should address two of the biggest sources of uncertainty in the present work, the 
contribution to the binding of the infrared region, and the variational approximation.

We have also been able to estimate the perturbative threebody force to be small. This is crucial information for the Faddeev formulation of the three-quark problem, since three-body forces are totally neglected there. At least we can now state that the error for the ground state, in the limit in which all three quarks are heavy, is modest and is of order $25 \mathrm{MeV}$ in the mass.

Acknowledgements Financial support by grants FPA2011-2785301, FIS2008-01323 plus 227431, Hadron-Physics2 (EU) and PR341856-BSCH, UCM-BSCH GR58/08, 910309, PR34/07-15875, SB2010-0012. We thankfully acknowledge the computer resources, technical expertise and assistance provided by the CeSViMa and the Spanish Supercomputing Network. FJLE is a recipient of a Caja Madrid fellowship and thanks the hospitality of the TU-Munich theoretical physics group and especially Nora Brambilla and Antonio Vairo, who have been influential in several aspects of this work.

\section{Appendix A: Static potential with BLM scale fixing}

In this section we introduce the Brodsky-Lepage-McKenzie scale fixing at NLO (that we have used in practical computations) as well as a sketch on how to proceed at NNLO (that we, however, have not further pursued).

\section{A.1 NLO potential}

The idea of the BLM renormalization scale choice [63] is to absorb the non-conformal terms proportional to the $\beta$ function of QCD into the running coupling. The resulting scale $\mu_{\mathrm{BLM}}$ is therefore fixed at NLO by demanding that the $\beta_{0}$ term cancels.

To one-loop order the idea can easily be carried out by shifting the scale of the strong coupling constant. This is done by substituting the solution of the renormalization group equation (with $\beta \simeq \beta_{0}$ taken at one-loop)

$$
\begin{aligned}
\alpha_{s}\left(\frac{1}{|\mathbf{r}|^{2}}\right) \simeq & \frac{\alpha_{s}\left(\mu_{\mathrm{BLM}}^{2}\right)}{1+\frac{\alpha_{s}\left(\mu_{\mathrm{BLM}}^{2}\right)}{4 \pi} \beta_{0} \log \left(\frac{1}{\left.\mu_{\mathrm{BLM}}^{2}|\mathbf{r}|^{2}\right)}\right)} \\
\simeq & \alpha_{s}\left(\mu_{\mathrm{BLM}}^{2}\right)+\frac{\alpha_{s}\left(\mu_{\mathrm{BLM}}^{2}\right)^{2}}{4 \pi} \beta_{0} \log \left(\mu_{\mathrm{BLM}}^{2}|\mathbf{r}|^{2}\right) \\
& +\cdots .
\end{aligned}
$$

Demanding therefore that

$$
\beta_{0}\left(\log \left(\mu_{\mathrm{BLM}}^{2}|\mathbf{r}|^{2}\right)+2 \gamma_{E}\right)=0
$$

we find that

$\mu_{\mathrm{BLM}}=\frac{e^{-\gamma_{E}}}{|\mathbf{r}|} \simeq 0.56 \frac{1}{|\mathbf{r}|}$
For charmonium, one would estimate numerically that

$\frac{1}{|\mathbf{r}|} \simeq \frac{1}{a_{c}}=\frac{m_{c} c^{2} \alpha_{s}}{\hbar c} \simeq 0.75 \mathrm{GeV}$

with the BLM scale correspondingly smaller, about $400 \mathrm{MeV}$ as is usual.

In terms of this scale, the potential to NLO takes the simpler form

$V_{\mathrm{LO}}^{(0)}+V_{\mathrm{NLO}}^{(0)}=-\frac{4}{3} \frac{\alpha_{s}\left(\mu_{\mathrm{BLM}}^{2}\right)}{r}\left(1+a_{1} \frac{\alpha_{s}\left(\mu_{\mathrm{BLM}}^{2}\right)}{4 \pi}\right)$.

\section{A.2 NNLO potential}

The NNLO contribution to the static potential however continues depending on $\beta_{0}$ and $\beta_{1}$

$$
\begin{aligned}
V^{(0)} \simeq & -\frac{4}{3} \frac{\alpha_{s}\left(\mu_{\mathrm{BLM}}^{2}\right)}{r}\left(1+a_{1} \frac{\alpha_{s}\left(\mu_{\mathrm{BLM}}^{2}\right)}{4 \pi}\right. \\
& \left.+\frac{\alpha_{s}^{2}\left(\mu_{\mathrm{BLM}}^{2}\right)}{(4 \pi)^{2}}\left(a_{2}+2 \beta_{1} \gamma_{E}+\beta_{0}^{2}\left(\frac{\pi^{2}}{3}-4 \gamma_{E}^{2}\right)\right)\right) .
\end{aligned}
$$

To eliminate these dependencies one needs to fix the BLM scale at two loops. However the expansion of the running coupling at the next order is significantly more difficult than Eq. (A.1). Then, to two loops, one has

$$
\begin{aligned}
\alpha_{s}^{\mathrm{NNLO}}\left(Q^{2}\right)= & \alpha_{s}^{\mathrm{NLO}}\left(Q^{2}\right) \\
& -b^{\prime} \alpha_{s}^{\mathrm{NLO}}\left(Q^{2}\right)^{2} \log \left(\log \left(\frac{Q^{2}}{\Lambda^{2}}\right)\right)
\end{aligned}
$$

where

$b^{\prime}=\frac{\beta_{1}}{4 \pi \beta_{0}}=\frac{153-19 N_{f}}{2 \pi\left(33-2 N_{f}\right)}$.

Since the double logarithm will yield a transcendental equation, one would have to fix the scale numerically. The computer algorithm proceeds as follows.

1. Fix the BLM scale analytically at NLO as in Eq. (A.2) above. Then $\mu_{\mathrm{BLM}}$ and $\alpha_{s}^{\mathrm{NLO}}\left(\mu_{\mathrm{BLM}}^{2}\right)$ are given.

2. Obtain $\Lambda$ from Eq. (19).

3. Obtain $\mu_{\mathrm{BLM}}^{\mathrm{NNLO}} 2$ from Eq. (A.5) by substituting it for $Q^{2}$ there. The equation relates $\mu_{\mathrm{BLM}}^{\mathrm{NNLO}}$ with $\mu_{\mathrm{BLM}}^{\mathrm{NLO}}$ and the dependency has to be solved for at the same time that one tries to make vanish all $\beta_{0}$ and $\beta_{1}$-proportional terms in the potential. This is best performed by an iterative Newton method.

We have found this unpractical for the time being and have only pursued the BLM method at NLO. 


\section{Appendix B: Numerical methods}

\section{B.1 Fourier transform}

To numerically transform potentials between momentum and coordinate space we employ a standard fast Fourier transform algorithm that implements the discrete formula

$A_{i}=\sum_{j=1}^{N} \exp \left(\frac{2 \pi i}{N}(i-1)(j-1)\right) \hat{A}_{j}$.

The wanted continuous transform is

$V(r)=\int \frac{d^{3} q}{(2 \pi)^{3}} e^{i \mathbf{q} \cdot \mathbf{r}} \hat{V}(|\mathbf{q}|)$

that after performing the angular integrals and grouping terms becomes

$V(r)=\frac{-2}{(2 \pi)^{2} r} \operatorname{Re}\left(i \int_{0}^{\infty} q d q \hat{V}(q) e^{i q r}\right)$.

We discretize a momentum interval $(\epsilon, \Lambda)$ where $\Lambda \simeq$ $50 \mathrm{GeV}$ is well above the quark mass scale (the hard scale) and $\epsilon$ is of order $(20 \mathrm{fm})^{-1}$, well below any soft scale in the problems treated. The momentum variable is then stepped linearly according to $q_{j}=\frac{\Lambda-\epsilon}{N} j$. The conjugate coordinate variable is automatically discretized as $r_{n}=2 \pi n /(\Lambda-\epsilon)$.

With these choices the vectors that appear in Eq. (B.1) are

$$
\begin{aligned}
& \hat{A}_{j}=\frac{(\Lambda-\epsilon)^{2}}{N^{2}} j \exp \left(\frac{2 \pi i}{N}(j-1)\right) \hat{V}\left(q_{j}\right), \\
& V_{\Lambda, \epsilon}\left(r_{l}\right)=\frac{-1}{2 \pi^{2} r_{l}} \mathfrak{R}\left(i \exp \left(-\frac{2 \pi i}{N} l\right) A_{l}\right) .
\end{aligned}
$$

\section{B.2 Minimization}

We have written a computer programme that employs the well-known Minuit minimization package from CERN [64] to fix the values of $\alpha_{s}, m_{c}$, and $m_{b}$ with the best possible description of the observables that we have selected. The Schroedinger equation for the reduced particle is solved quasi-exactly (on a computer) with the perturbative potential to LO, NLO and NNLO, for both charm and bottom quarks. This is performed by discretizing the second derivative of the reduced radial function with the symmetric formula

$u^{\prime \prime}\left(r_{i}\right) \simeq \frac{u\left(r_{i+1}\right)+u\left(r_{i-1}\right)-2 u\left(r_{i}\right)}{h^{2}}$

After reducing the Hamiltonian to a numerical matrix, this is diagonalized. Since the radial problem is one-dimensional, accuracy in the diagonalization is not an issue.

In the LO evaluation of the potential the coupling constant is fixed at a renormalization scale $\mu^{2}=m_{c}^{2}$ or $\mu^{2}=m_{b}^{2}$ employing the NLO running. Except for this small modification (needed since the same coupling constant cannot sensibly be used in both charmonium and bottomonium systems), the computation is perfectly consistent with perturbation theory, so that at NNLO, in the pure NNLO potential pieces the LO coupling constant is employed, whereas in the LO piece the NNLO coupling constant features, and so forth. As far as we can imagine no contamination is introduced from higher pieces in perturbation theory. We have also employed the perturbative formulas for $\Gamma_{J / \Psi \rightarrow \eta_{c}}$.

We have of course checked the sensitivity of the numerical results to the number of points used in the grid solving Schroedinger's equation (300 turns out to yield very precise answers for low-lying states in the respective potentials), the maximum size of the grid (that extends to $4 \mathrm{fm}$ and beyond) and other numeric artifacts.

\section{B.3 Iterative scale determination}

When the argument of the coupling constant depends on the coupling constant itself such as $\alpha_{s}=\bar{\alpha}_{s}\left(m \alpha_{s}\right)$ an iterative method is in order.

We employ Newton's iterative numerical method. Denoting $\alpha_{s}^{(n)}$ as the successive approximations to the numeric value, and $\tilde{\alpha}_{s}$ the function in Eq. (A.5) or similar, then the coupling constant has been found when

$F\left(\alpha_{s}\right) \equiv \tilde{\alpha}_{s}\left(\frac{M_{J / \psi}}{4} \alpha_{s}\right)-\alpha_{s}=0$.

Newton's iteration, as long as $F \neq 0$ with sufficient significance, is given by

$\alpha_{s}^{(n)}=\alpha_{s}^{(n-1)}-\frac{F\left(\alpha_{s}^{(n-1)}\right)}{F^{\prime}\left(\alpha_{s}^{(n-1)}\right)}$.

One can take as initial guess $\alpha_{s}^{0}=\tilde{\alpha}_{s}\left(\mu^{2}\right)$ at any standard renormalization scale, and then iterate Eq. (B.6).

As an alternative we also employ Jacobi's fixed point method, in which one starts with a guess $\alpha_{s}^{(0)}$ (presumably) larger than the true value, and then iterate the recursive relation $\alpha_{s}^{(n)}=\tilde{\alpha}_{s}\left(\frac{M_{J / \psi}}{4} \alpha_{s}^{(0)}\right)$ until convergence.

\section{B.4 Monte Carlo computation of three-body matrix elements}

The variational matrix elements in the baryon computation are multidimensional integrals. Three particles, after center of mass separation, require six momentum integrations in the kinetic energy evaluation. Two-body potentials add one loop to the matrix element, up to nine dimensions. In the three-body force computation there are two exchanged momenta, and thus twelve-dimensional integrals. We make no attempt at separating rigid rotations and evaluate all 
these matrix elements numerically employing the Vegas algorithm [65-67].

In the matrix element

$$
\frac{\langle\psi|H| \psi\rangle}{\langle\psi \mid \psi\rangle}
$$

we compute both numerator and denominator (the wavefunction normalization) numerically. This makes the limits of integration quite irrelevant for the computation since the function is normalized to one in the same region where the Hamiltonian matrix element is computed, so that no probability density is missed. In practice we never extend integration beyond the hard scale $m_{c}$ or $m_{b}$ (one expects the variational parameters $\alpha_{\rho}$ and $\alpha_{\lambda}$ to concentrate the momentum wave unction around the soft scale $\alpha_{s} m_{c}$ or $\alpha_{s} m_{b}$ ).

We employ a minimum of seven million evaluations of the Hamiltonian and reach a precision of about $10 \mathrm{MeV}$ for standard computations, increasing this as needed. A $3 \mathrm{GHz}$ processor can swipe an $8 \times 8$ set of variational parameters $\alpha_{\rho}, \alpha_{\lambda}$ in about an hour.

Our program performs wave function symmetrization (or mixed symmetrization for the $c c b$ and $b b c$ systems) by invoking the wave function with exchanged spins and momentum arguments as needed. Although here we have not taken spin corrections into account, since they are unknown for the three-body problem, our program is performing (trivial) spin sums to allow for a simple upgrade once the spin kernels for triply heavy baryons become available.

\section{References}

1. T. Melde, W. Plessas, B. Sengl, Phys. Rev. D 77, 114002 (2008). arXiv:0806.1454 [hep-ph]

2. U. Loring, B.C. Metsch, H.R. Petry, Eur. Phys. J. A 10, 395-446 (2001). hep-ph/0103289

3. L.Y. Glozman, D.O. Riska, hep-ph/9412231

4. N. Brambilla, S. Eidelman, B.K. Heltsley, R. Vogt, G.T. Bodwin, E. Eichten, A.D. Frawley, A.B. Meyer et al., Eur. Phys. J. C 71, 1534 (2011). arXiv:1010.5827 [hep-ph]

5. N. Brambilla et al. (Quarkonium Working Group Collaboration), hep-ph/0412158

6. N. Brambilla, A. Pineda, J. Soto, A. Vairo, Rev. Mod. Phys. 77, 1423 (2005). hep-ph/0410047

7. N. Brambilla, A. Pineda, J. Soto, A. Vairo, Phys. Rev. D 63, 014023 (2001). hep-ph/0002250

8. A. Pineda, A. Vairo, Phys. Rev. D 63, 054007 (2001). hep-ph/ 0009145

9. N. Brambilla, A. Pineda, J. Soto, A. Vairo, Nucl. Phys. B 566, 275 (2000). hep-ph/9907240

10. N. Brambilla, J. Ghiglieri, A. Vairo, Phys. Rev. D 81, 054031 (2010). arXiv:0911.3541 [hep-ph]

11. A. Vairo, in Proceedings of Quark Confinement and the Hadron Spectrum IV, ed. by W. Lucha, K. Maung Maung (World Scientific, Singapore, 2001). Eprint hep-ph/0010191

12. Y. Schroder, Phys. Lett. B 447, 321-326 (1999). arXiv:hep-ph/ 9812205 [hep-ph]

13. M. Peter, Nucl. Phys. B 501, 471-494 (1997). hep-ph/9702245
14. P. Watson, H. Reinhardt, Phys. Rev. D 76, 125016 (2007). arXiv:0709.0140 [hep-th]

15. C. Popovici, P. Watson, H. Reinhardt, AIP Conf. Proc. 1343, 373 375 (2011). arXiv:1011.2151 [hep-ph]

16. R. Barbieri, M. Ciafaloni, P. Menotti, Nuovo Cimento A 55, 701723 (1968)

17. A. Laschka, N. Kaiser, W. Weise, Phys. Rev. D 83, 094002 (2011). arXiv:1102.0945 [hep-ph]

18. A. Laschka, N. Kaiser, W. Weise, in Proceedings of the XIV International Conference on Hadron Spectroscopy (hadron2011), 13 17 June 2011, Munich, Germany (2011). arXiv:1108.5591 [hep$\mathrm{ph}]$

19. B.A. Kniehl, A.A. Penin, M. Steinhauser, V.A. Smirnov, Phys. Rev. D 65, 091503 (2002). arXiv:hep-ph/0106135 [hep-ph]

20. G. Altarelli, in Proceedings of PASI (2002). hep-ph/0204179

21. S. Bethke, Eur. Phys. J. C 64, 689-703 (2009). arXiv:0908.1135 [hep-ph]

22. S. Bethke, A.H. Hoang, S. Kluth, J. Schieck, I.W. Stewart, S. Aoki, M. Beneke, J. Blumlein et al., Workshop on Precision Measurements of alphas. arXiv:1110.0016 [hep-ph]

23. G. Rodrigo, A. Santamaria, Phys. Lett. B 313, 441 (1993). hep-ph/9305305

24. N. Brambilla, X. Garcia i Tormo, J. Soto, A. Vairo, Phys. Rev. D 75, 074014 (2007). hep-ph/0702079

25. P. Guo, A.P. Szczepaniak, G. Galata, A. Vassallo, E. Santopinto, Phys. Rev. D 77, 056005 (2008). arXiv:0707.3156 [hep-ph]

26. C. Alexandrou, P. de Forcrand, O. Jahn, Nucl. Phys. B, Proc. Suppl. 119, 667-669 (2003). hep-lat/0209062

27. A.S. Bakry, D.B. Leinweber, A.G. Williams, Gluonic profile of the static baryon at finite temperature. arXiv:1107.0150 [hep-lat]

28. N. Brambilla, A. Pineda, J. Soto, A. Vairo, Phys. Rev. D 60, 091502 (1999). hep-ph/9903355

29. A.H. Hoang, Phys. Rev. D 59, 014039 (1999). hep-ph/9803454

30. A. Pineda, $\mathrm{PhD}$ dissertation presented to the Univ. of Barcelona (1998)

31. M. Beneke, Phys. Lett. B 434, 115-125 (1998). hep-ph/9804241

32. K.G. Chetyrkin, M. Steinhauser, Phys. Rev. Lett. 83, 4001-4004 (1999). hep-ph/9907509

33. J.-L. Domenech-Garret, M.-A. Sanchis-Lozano, Phys. Lett. B 669, 52-57 (2008). arXiv:0805.2916 [hep-ph]

34. K. Nakamura et al. (Particle Data Group Collaboration), J. Phys. G 37, 075021 (2010)

35. N. Brambilla, Y. Jia, A. Vairo, Phys. Rev. D 73, 054005 (2006). hep-ph/0512369

36. B. Blossier et al. (ETM Collaboration), Phys. Rev. D 82, 114513 (2010). arXiv:1010.3659 [hep-lat]

37. A. Pich, arXiv:1107.1123 [hep-ph]

38. K.G. Chetyrkin, A. Retey, Nucl. Phys. B 583, 3-34 (2000). hep-ph/9910332

39. J.-R. Zhang, M.-Q. Huang, Phys. Lett. B 674, 28-35 (2009). arXiv:0902.3297 [hep-ph]

40. Yu Jia, J. High Energy Phys. 0610, 073 (2006). hep-ph/0607290

41. J.M. Flynn, E. Hernandez, J. Nieves, arXiv:1110.2962 [hep-ph]

42. B. Silvestre-Brac, Few-Body Syst. 20, 1-25 (1996)

43. V. Hasenfratz et al., Phys. Lett. B 94, 401 (1980)

44. S. Nussinov, Phys. Rev. Lett. 52, 966 (1984)

45. S. Nussinov, Phys. Rev. Lett. 51, 2081 (1983)

46. R. Alkofer, C.S. Fischer, F.J. Llanes-Estrada, K. Schwenzer, Ann. Phys. 324, 106-172 (2009). arXiv:0804.3042 [hep-ph]

47. J.D. Bjorken, Is the CCC a new deal for baryon spectroscopy?, in FERMILAB-CONF-85-069, C85-04-20 (Apr 1985)

48. W. Roberts, M. Pervin, Int. J. Mod. Phys. A 23, 2817-2860 (2008). arXiv:0711.2492 [nucl-th]

49. J. Vijande et al., Phys. Rev. D 70, 054022 (2004)

50. A.P. Martynenko, Phys. Lett. B 663, 317-321 (2008). arXiv: 0708.2033 [hep-ph] 
51. S. Migura, D. Merten, B. Metsch, H.-R. Petry, Eur. Phys. J. A 28, 41 (2006). hep-ph/0602153

52. B. Patel, A. Majethiya, P.C. Vinodkumar, Pramana 72, 679-688 (2009). arXiv:0808.2880 [hep-ph]

53. S. Meinel, Prediction of the $\Omega_{b b b}$ mass from lattice QCD. Phys. Rev. D 82, 114514 (2010). The $\Omega_{c c c}$ data was reported by C. McNeile, communication to the VIIIth meeting of the Quarkonium Working Group, GSI, Darmstadt, October 4-7, 2011 (unpublished)

54. X.-H. Guo, K.-W. Wei, X.-H. Wu, Phys. Rev. D 78, 056005 (2008). arXiv:0809.1702 [hep-ph]

55. N. Brambilla, P. Consoli, G.M. Prosperi, Phys. Rev. D 50, 5878 (1994). hep-th/9401051

56. N. Brambilla, A. Vairo, T. Rosch, Phys. Rev. D 72, 034021 (2005). hep-ph/0506065

57. F.J. Llanes-Estrada, G.M. Navarro, arXiv:1108.1859 [nucl-th]

58. P. Bicudo, M. Cardoso, T. Van Cauteren, F.J. Llanes-Estrada, Phys. Rev. Lett. 103, 092003 (2009). arXiv:0902.3613 [hep-ph]
59. Y.Q. Chen, S.-Z. Wu, arXiv:1106.0193

60. S.P. Baranov, V.L. Slad, Phys. At. Nucl. 67, 808-814 (2004). hep-ph/0603090

61. A. Austregesilo (COMPASS Collaboration), J. Phys. Conf. Ser. 312, 032006 (2011). arXiv:1109.0230 [hep-ex]

62. L. Schmitt, S. Paul, R. Kuhn, M.A. Moinester, hep-ex/0310049

63. S.J. Brodsky, G.P. Lepage, P.B. Mackenzie, Phys. Rev. D 28, 228 (1983)

64. F. James, M. Roos, Comput. Phys. Commun. 10, 343-367 (1975)

65. G.P. Lepage, Cornell preprint CLNS 80-447, March 1980

66. G.P. Lepage, J. Comput. Phys. 27, 192-203 (1978)

67. T. Hahn, Comput. Phys. Commun. 168, 78-95 (2005). hep-ph/ 0404043

68. A.H. Hoang, A. Jain, I. Scimemi, I.W. Stewart, Phys. Rev. D 82, 011501 (2010). arXiv:0908.3189 [hep-ph] 